Homology, Homotopy and Applications, vol.15(1), 2013, pp.101-126

\title{
ON THE HOMOLOGY OF THE SPACE OF SINGULAR KNOTS
}

\author{
HOSSEIN ABBASPOUR AND DAVID CHATAUR
}

(communicated by Claude Cibils)

\begin{abstract}
In this paper we introduce various associative products on the homology of the space of knots and singular knots in $S^{n}$. We prove that these products are related through a desingularization map. We also compute some of these products and prove the non-triviality of the desingularization morphism. Using direct computations, we prove that some of these products are indeed commutative.
\end{abstract}

\section{Introduction}

Various authors have tried to introduce a general framework for the structures introduced by Chas-Sullivan [CS1] on the homology and on the equivariant homology of the free loop space of a closed manifold. One approach, first used implicitly in $[\mathbf{K S}]$ and then formulated in $[\mathbf{G S}]$, is the notion of fibrewise (homotopy) monoid. This formulation is inspired by Cohen-Jones' $[\mathbf{C J}]$ homotopy theoretic description of the Chas-Sullivan product using ring spectra. Laudenbach's formulation [Lau using transverse chain bicomplex can also be easily adapted to the fibrewise monoid setting and is sufficiently efficient for geometric calculation. In this article we apply Budney's [B1], Gruher-Salvatore's [GS] and Salvatore's [S] results to introduce and calculate various algebra structures on the homology of knots, immersions, and singular knots with $k$ double points. Moreover, we prove that these structures are related through some naturally defined maps and a desingularization morphism that we define.

Let $\operatorname{Imm}\left(S^{1}, S^{n}\right)$ be the space of all immersions of $S^{1}$ in $S^{n}$. Then $\operatorname{Imm}^{\prime}\left(S^{1}, S^{n}\right)$ is defined to be the space of immersions $\gamma: S^{1} \rightarrow S^{n}$ with no singularity at the marked point $\operatorname{ev}_{0}(\gamma):=\gamma(0)$.

The space of knots $\operatorname{Emb}\left(S^{1}, S^{n}\right)$ is the space of all embeddings of $S^{1}$ in $S^{n}$. We will introduce various associative algebra structures on the homology of $\operatorname{Emb}\left(S^{1}, S^{n}\right)$, $\operatorname{Imm}^{\prime}\left(S^{1}, S^{n}\right)$ and $\operatorname{Imm}\left(S^{1}, S^{n}\right)$, and finally $\coprod_{k \in \mathbb{N}} \operatorname{Imm}_{k}{ }_{k}\left(S^{1}, S^{n}\right)$ the space of singular knots with a finite number of transverse double points away from the marked point and no other singular points. Here $k$ stands for the number of double points.

The second author is partially supported by ANR grant 06-JCJC-0042 "Opérades, Bigèbres et Théories d'Homotopie".

Received April 25, 2012, revised November 9, 2012; published on April 11, 2013.

2000 Mathematics Subject Classification: 55P50, 55N45, 57Q45.

Key words and phrases: knot, singular knot, long knot, free loop space, string operation, chord diagram.

Article available at http://intlpress.com/HHA/v15/n1/a6 and doi:10.4310/HHA.2013.v15.n1.a6

Copyright (C) 2013, International Press. Permission to copy for private use granted. 
In order to introduce these products, we use the fact that each of these spaces is homotopy equivalent to the total space of a fibration whose fibres form a continuous family of homotopy associative monoids, and the base space is a closed oriented manifold. The archetype of such fibrations is the loop space fibration $\Omega M \rightarrow L M$ $\rightarrow M$ of a closed oriented manifold. Here $L M=C^{\infty}\left(S^{1}, M\right)$ and $\Omega M$ are respectively the free loop space and based loop space of $M$. The (classical based) loop spaces have a homotopy associative product, but one also has the option of considering Moore (based) loop spaces (Example 3.2) whose concatenation products are strictly associative. We then prove that

Theorem 3.3. The graded group $H_{*+2 n-1}\left(\operatorname{Emb}\left(S^{1}, S^{n}\right)\right)$ can be equipped with a graded commutative and associative product $\mu_{\mathrm{em}}(-,-)$, which is compatible with the intersection product on $H_{*+2 n-1}\left(U S^{n}\right)$ via the map $\mathrm{ev}_{*}: H_{*}\left(\operatorname{Emb}\left(S^{1}, S^{n}\right)\right) \rightarrow$ $H_{*}\left(U S^{n}\right)$. Here $U S^{n}$ is the unit sphere bundle of the tangent bundle of $S^{n}$, and $\mathrm{ev} i s$ the map induced by ev: $\operatorname{Emb}\left(S^{1}, S^{n}\right) \rightarrow U S^{n}$,

$$
\operatorname{ev}(\gamma)=\left(\gamma(0), \gamma^{\prime}(0) /\left\|\gamma^{\prime}(0)\right\|\right)
$$

which sends a knot to the normal tangent vector at $t=0$.

Similarly, we prove that

Theorem 3.4. The graded group $H_{*+2 n-1}\left(\operatorname{Imm}^{\prime}\left(S^{1}, S^{n}\right)\right)$ can be equipped with a graded commutative and associative product $\mu_{\mathrm{im}}(-,-)$, which is compatible with the intersection product on $H_{*+2 n-1}\left(U S^{n}\right)$ via the map induced by ev: $\operatorname{Imm}^{\prime}\left(S^{1}, S^{n}\right) \rightarrow$ $U S^{n}$.

At the first sight it is not obvious if this algebra is commutative because the product on the fibres of the fibration ev: $\operatorname{Imm}^{\prime}\left(S^{1}, S^{n}\right) \rightarrow U S^{n}$ is not a priori commutative (even up to homotopy). The commutativity of the product follows from some explicit computations in the last section using spectral sequences (see Theorem 6.2). After restricting to the subspaces $\operatorname{Imm}_{k}{ }_{k}\left(S^{1}, S^{n}\right)$ 's, one obtains a collection of maps

$$
\begin{aligned}
\mu_{\mathrm{im}}^{k, l}(-,-)=H_{*}\left(\operatorname{Imm}_{k}{ }_{k}\left(S^{1}, S^{n}\right)\right) \otimes H_{*}\left(\operatorname{Imm}_{l}{ }_{l}\left(S^{1}, S^{n}\right)\right) & \\
& \rightarrow H_{*-2 n+1}\left(\operatorname{Imm}_{k+l}{ }_{k+l}\left(S^{1}, S^{n}\right)\right),
\end{aligned}
$$

which is compatible in the following sense:

$$
\mu_{\mathrm{im}}^{k+l, m}\left(\mu_{\mathrm{im}}^{k, l}(a, b), c\right)=\mu_{\mathrm{im}}^{k, l+m}\left(a, \mu_{\mathrm{im}}^{l, m}(b, c)\right) .
$$

In order to compare the algebra structures on the homologies of knot and singular knot spaces, we introduce a desingularization map. Informally speaking, we resolve a singular knot at a double point in all possible ways, parametrized by the unit vectors perpendicular to the tangent plane at the singularity. Of course this map has a certain degree, and it is not a map of algebras. However, it verifies some natural compatibility conditions with respect to the number of singularities and the product. This is stated in the main theorem of this article as follows:

Theorem 5.4. The desingularization morphisms

$$
\phi_{k}: H_{*}\left(\operatorname{Imm}_{k}^{\prime}\left(S^{1}, S^{n}\right)\right) \rightarrow H_{*+k(n-3)}\left(\operatorname{Emb}\left(S^{1}, S^{n}\right)\right),
$$

$k \geqslant 0$ are compatible with the multiplicative structures, i.e., for $x \in H_{*}\left(\operatorname{Imm}_{k}{ }_{k}\left(S^{1}, S^{n}\right)\right)$ 
and $y \in H_{*}\left(\operatorname{Imm}_{l}{ }_{l}\left(S^{1}, S^{n}\right)\right)$,

$$
\mu_{\mathrm{em}}\left(\phi_{k}(x), \phi_{l}(y)\right)=\phi_{k+l}\left(\mu_{\mathrm{im}}^{k, l}(x, y)\right) .
$$

In other words,

$$
\phi=\oplus \phi_{k}: \bigoplus_{k} \mathbb{H}_{*}\left(\operatorname{Imm}_{k}^{\prime}\left(S^{1}, S^{n}\right)\right) \rightarrow \mathbb{H}_{*}\left(\operatorname{Emb}\left(S^{1}, S^{n}\right)\right)
$$

is a map of algebras, where

$$
\mathbb{H}_{*}\left(\operatorname{Imm}_{k}^{\prime}\left(S^{1}, S^{n}\right)\right)=H_{*+2 n-1}\left(\operatorname{Imm}_{k}^{\prime}\left(S^{1}, S^{n}\right)\right)
$$

and

$$
\mathbb{H}_{*}\left(\operatorname{Emb}\left(S^{1}, S^{n}\right)\right)=H_{*+2 n-1}\left(\operatorname{Emb}\left(S^{1}, S^{n}\right)\right) .
$$

In the case of singular knots with finitely-many transverse singularities, one naturally expects a compatibility with the Vassiliev spectral sequences. This issue is not addressed in this paper and merits further investigation.

\section{Conventions}

Here $S^{n}$ is the unit sphere in $\mathbb{R}^{n+1}$, and we make the identification $\mathbb{R}^{n} \simeq\{0\} \times$

$\mathbb{R}^{n} \subset \mathbb{R}^{n+1}$. We think of $S O(n) \subset S O(n+1)$ as the stabilizer of $(1,0, \ldots, 0)$ and $S O(n-1) \subset S O(n+1)$ as the stabilizer of $(1,0, \ldots, 0)$ and the tangent vector $(0,1,0, \ldots, 0) \in T_{(1,0, \ldots, 0)} S^{n}$. As a consequence, $S O(n+1) / S O(n-1)$ is identified with $U S^{n}$ as the unit tangent sphere bundle of $S^{n}$. For a manifold $M, L M=$ $C^{\infty}\left(S^{1}, M\right)$ is the free loop space and $\mathrm{ev}_{0}: L M \rightarrow M$ maps a loop $\gamma: S^{1} \rightarrow M$ to its marked point $\operatorname{ev}_{0}(\gamma):=\gamma(0)$. By abuse of notation, we denote the induced map in homology also by $\mathrm{ev}_{0}$. If $M$ is a $d$-dimensional closed oriented manifold, then let $\mathbb{H}_{*}(M)=H_{*+d}(M, \mathbb{Z})$ be the regraded homology of $M$, which is a commutative and associative algebra once it is equipped with the intersection product. Similarly, $\mathbb{H}_{*}(L M, \mathbb{Z})=H_{*+d}(L M, \mathbb{Z})$ is the homology of $L M$ with a shift in degree, which is a graded commutative and associative algebra once it is equipped with the ChasSullivan loop product.

\section{Acknowledgements}

The first author was partially supported by the Hausdorff Research Institute when this paper was written. He also thanks Ryan Budney and François Laudenbach for some helpful communications.

The second author thanks the Laboratoire Jean Leray at the University of Nantes for their invitation through the Matpyl program.

\section{Immersions and knots}

In this section we collect a few well-known facts about various immersion and embedding spaces and their homotopy types. Note that all immersion and embedding spaces are equipped with the induced compact-open $C^{\infty}$ (weak) topology ([Ada, Hir $]$ ). This is the smallest topology that makes all jet maps $J: C^{\infty}\left(S^{1}, M\right) \rightarrow$ $C^{0}\left(S^{1}, J^{k}\left(S^{1}, M\right)\right)$ or $J: C^{\infty}(\mathbb{R}, M) \rightarrow C^{0}\left(\mathbb{R}, J^{k}(\mathbb{R}, M)\right), k=0,1, \ldots$ continuous. 
The choice of $\mathbb{R}$ or $S^{1}$ depends on whether we are working with long immersions/embeddings or compact ones. By the space of long immersions we mean

$$
\begin{array}{r}
\operatorname{Imm}^{l}\left(\mathbb{R}, \mathbb{R}^{n}\right)=\left\{f: \mathbb{R} \rightarrow \mathbb{R}^{n} \text { smooth immersion, } \operatorname{supp}(f)\right. \\
\left.\subset(-1,1) \text { and } f([-1,1]) \subset B^{n}\right\},
\end{array}
$$

where $\operatorname{supp}(f)=\left\{t \in \mathbb{R} \subset \mathbb{R}^{n} \mid f(t) \neq(t, 0, \ldots, 0)\right\}$ and $B^{n}$ is the unit ball in $\mathbb{R}^{n}$.

An important subspace of $\operatorname{Imm}^{l}\left(\mathbb{R}, \mathbb{R}^{n}\right)$ is $\operatorname{Imm}_{k}^{l}\left(\mathbb{R}, \mathbb{R}^{n}\right)$, the space of singular knots with transverse double points. These are the immersions with only double transversal self-intersections.

Remark 2.1. One naturally asks if we get interesting subspaces of $\operatorname{Imm}^{l}\left(\mathbb{R}, \mathbb{R}^{n}\right)$ by relaxing the transversality condition for self-intersections imposed on the elements of $\operatorname{Imm}_{k}^{l}\left(\mathbb{R}, \mathbb{R}^{n}\right)$. For example, one can also consider the space $\operatorname{Emb}_{\text {sing }}^{l}\left(\mathbb{R}, \mathbb{R}^{n}\right)$ as long singular knots consisting of the long immersions $f: \mathbb{R} \rightarrow \mathbb{R}^{n}$, which are embeddings outside of a finite set $A \subset[-1,1] \subset \mathbb{R}$. We additionally require that for all pairs $x_{i}$ and $x_{j} \in A$ with $f\left(x_{i}\right)=f\left(x_{j}\right)$, the corresponding branches of the knots have finite order tangency. We have that

$$
\coprod_{k \in \mathbb{N}} \operatorname{Imm}_{k}^{l}\left(\mathbb{R}, \mathbb{R}^{n}\right) \subset \operatorname{Emb}_{\text {sing }}^{l}\left(\mathbb{R}, \mathbb{R}^{n}\right) \subset \operatorname{Imm}^{l}\left(\mathbb{R}, \mathbb{R}^{n}\right),
$$

but it turns out that $\operatorname{Emb}_{\text {sing }}^{l}\left(\mathbb{R}, \mathbb{R}^{n}\right)$ has the same homotopy type as $\operatorname{Imm}^{l}\left(\mathbb{R}, \mathbb{R}^{n}\right)$. In short the complement of an infinite codimension closed subspace of $\operatorname{Imm}^{l}\left(\mathbb{R}, \mathbb{R}^{n}\right)$ has the same homotopy type as $\operatorname{Imm}^{l}\left(\mathbb{R}, \mathbb{R}^{n}\right)$. This is the content of Lemma 3.2.1 in $[\mathbf{B r}]$.

Another important space in this article is the space of long knots $\operatorname{Emb}^{l}\left(\mathbb{R}, \mathbb{R}^{n}\right)$, which is the subspace of $\operatorname{Imm}^{l}\left(\mathbb{R}, \mathbb{R}^{n}\right)$ consisting of embeddings. The space of long knots $\operatorname{Emb}^{l}\left(\mathbb{R}, \mathbb{R}^{n}\right)$ is interesting on its own and has been the subject of many recent works. It was studied, in particular, by Vassiliev [Va] in the context of finite type invariants. Budney $[\mathbf{B} \mathbf{1}]$ recently proved that there is an action of the little disc operad on the space of framed long knots in $\mathbb{R}^{n}$ which, in the particular case of $n=3$, induces an action on a subspace of the framed long knots, homotopy equivalent to the space of (unframed) long knots. The latter provides us with a deep understanding of the homotopy type of the space of long knots in dimension 3 [B2]. It is noteworthy to mention that this action was predicted by Tourchine [Tor], who had proved that the $E^{2}$-page of Vassiliev's spectral sequence has a Gerstenhaber structure, which is part of the structure induced by the action of the little disk operad. More recently, Arone, Lambrechts, Tourtchine and Volć [ALTV] have computed the rational homology (in terms of graph complexes) by proving that the Vassiliev spectral sequence collapses at the $E_{1}$-term when $n \geqslant 4$.

The reason we are interested in $\operatorname{Emb}^{l}\left(\mathbb{R}, \mathbb{R}^{n}\right)$ is that it recovers via a Borel construction, the space of knots in $S^{n}$ as we will explain henceforth. There is an action of $S O(n-1)$ on $\operatorname{Emb}^{l}\left(\mathbb{R}, \mathbb{R}^{n}\right)$ via the identification $\mathbb{R}^{n}=\mathbb{R} \times \mathbb{R}^{n-1}$. These are the rotations around the fixed long axis. We also make the identification $\mathbb{R}^{n}=\{0\} \times \mathbb{R}^{n} \subset \mathbb{R}^{n+1}$, which provides us with a natural inclusion $S O(n-1) \hookrightarrow$ $S O(n+1)$, hence an action of $S O(n-1)$ on $S O(n+1)$. One then considers the Borel quotient $S O(n+1) \times{ }_{S O(n-1)} \operatorname{Emb}^{l}\left(\mathbb{R}, \mathbb{R}^{n}\right)$, which has the same homotopy type as 
$\operatorname{Emb}\left(S^{1}, S^{n}\right)$ (see [BC, Proposition 1.4]). A homotopy equivalence is given by the stereographic map with $(1,0, \ldots, 0) \in S^{n} \subset \mathbb{R}^{n+1}=\mathbb{R} \times \mathbb{R}^{n}$ as the center. Given a pair

$$
(A, \gamma) \in S O(n+1) \times_{S O(n-1)} \operatorname{Emb}^{l}\left(\mathbb{R}, \mathbb{R}^{n}\right),
$$

one can compactify $\gamma$ in $\mathbb{R}^{n+1}$ to get a knot in $S^{n} \subset \mathbb{R}^{n+1}$ passing through $(1,0, \ldots, 0)$. Then we let $A$ act on $\gamma$ to get an element of $\operatorname{Emb}\left(S^{1}, S^{n}\right)$. This defines a homotopy equivalence map

$$
\rho_{\mathrm{emb}}: S O(n+1) \times_{S O(n-1)} \operatorname{Emb}^{l}\left(\mathbb{R}, \mathbb{R}^{n}\right) \rightarrow \operatorname{Emb}\left(S^{1}, S^{n}\right),
$$

which makes the following diagram commute $[\mathbf{B C}]$ :

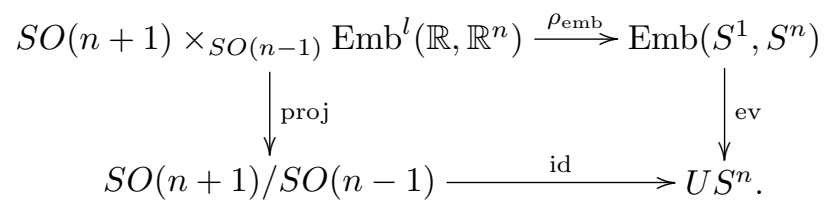

Here $\operatorname{ev}(\gamma)=\left(\gamma(0), \gamma^{\prime}(0) /\left\|\gamma^{\prime}(0)\right\|\right)$ for $\gamma \in \operatorname{Emb}\left(S^{1}, S^{n}\right)$, and the lower horizontal arrow is a diffeomorphism (see Conventions in the introduction). Therefore, we have the isomorphism

$$
\rho_{\mathrm{em}}: H_{*}\left(S O(n+1) \times_{S O(n-1)} \operatorname{Emb}^{l}\left(\mathbb{R}, \mathbb{R}^{n}\right)\right) \rightarrow H_{*}\left(\operatorname{Emb}\left(S^{1}, S^{n}\right)\right) .
$$

Similarly, one can consider the Borel construction $S O(n+1) \times{ }_{S O(n-1)} \operatorname{Imm}^{l}\left(\mathbb{R}, \mathbb{R}^{n}\right)$ and $S O(n+1) \times_{S O(n-1)} \operatorname{Imm}_{k}^{l}\left(\mathbb{R}, \mathbb{R}^{n}\right)$ and the maps of fibrations

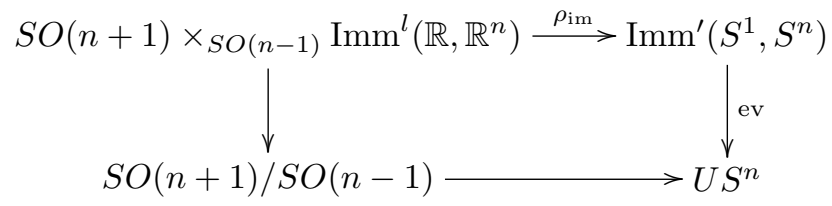

and

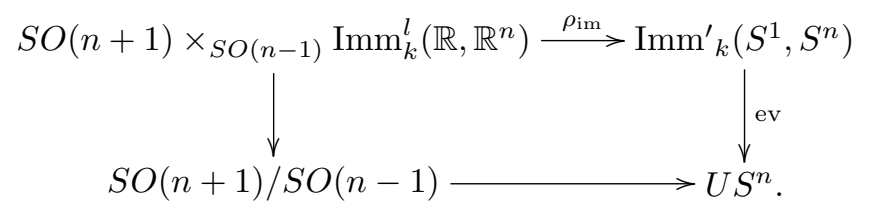

The homotopy equivalences induced by the stereographic map give rise to the isomorphisms

$$
\begin{aligned}
& \rho_{\mathrm{im}}: H_{*}\left(S O(n+1) \times_{S O(n-1)} \operatorname{Imm}^{l}\left(\mathbb{R}, \mathbb{R}^{n}\right)\right) \rightarrow H_{*}\left(\operatorname{Imm}^{\prime}\left(S^{1}, S^{n}\right)\right), \\
& \rho_{\mathrm{im}}^{k}: H_{*}\left(S O(n+1) \times_{S O(n-1)} \operatorname{Imm}_{k}^{l}\left(\mathbb{R}, \mathbb{R}^{n}\right)\right) \rightarrow H_{*}\left(\operatorname{Imm}_{k}{ }_{k}\left(S^{1}, S^{n}\right)\right) .
\end{aligned}
$$

We have the following sequence of inclusions:

$$
\begin{aligned}
& \operatorname{Emb}^{l}\left(\mathbb{R}, \mathbb{R}^{n}\right) \subset \operatorname{Imm}_{k}^{l}\left(\mathbb{R}, \mathbb{R}^{n}\right) \subset \operatorname{Imm}^{l}\left(\mathbb{R}, \mathbb{R}^{n}\right), \\
& \operatorname{Emb}\left(S^{1}, S^{n}\right) \subset \operatorname{Imm}_{k}^{\prime}\left(S^{1}, S^{n}\right) \subset \operatorname{Imm}^{\prime}\left(S^{1}, S^{n}\right) .
\end{aligned}
$$

The immersion spaces were widely studied in the 50's and 60 's. One of the most 


\begin{tabular}{|c|c|}
\hline $\operatorname{Emb}^{l}\left(\mathbb{R}, \mathbb{R}^{n}\right)$ & Long knots in $\mathbb{R}^{n}$ \\
\hline $\operatorname{Emb}\left(S^{1}, S^{n}\right)$ & Embeddings in $S^{n}($ Knots $)$ \\
\hline $\operatorname{Imm}^{l}\left(\mathbb{R}, \mathbb{R}^{n}\right)$ & Long immersions in $\mathbb{R}^{n}$ \\
\hline $\operatorname{Imm}^{\prime}\left(S^{1}, S^{n}\right)$ & Immersions in $S^{n}$ without singularity at $t=0$ \\
\hline $\operatorname{Imm}_{k}^{l}\left(\mathbb{R}, \mathbb{R}^{n}\right)$ & Long immersions in $\mathbb{R}^{n}$ with $k$ double points \\
\hline $\operatorname{Imm}^{\prime}{ }_{k}\left(S^{1}, S^{n}\right)$ & Immersions in $S^{n}$ with $k$ double points away from $t=0$ \\
\hline
\end{tabular}

Table 1: Notation

notable results is the Hirsch-Smale theorem. A special case of this theorem is as follows:

Theorem 2.2 (Hirsch [Hir]-Smale [Sm]). For a smooth manifold $M$, the 1-jet map $D: \operatorname{Imm}\left(S^{1}, M\right) \rightarrow L U M$, given by $D: \gamma \mapsto\left(\gamma, \gamma^{\prime} /\left\|\gamma^{\prime}\right\|\right)$, is a homotopy equivalence.

Here $U M$ is the unit sphere bundle of $M$. This theorem allows us to compute the homotopy type of some subspaces of the $\operatorname{Imm}\left(S^{1}, M\right)$. For instance, let $\operatorname{Imm}_{*, v}\left(S^{1}, M\right)$ be the subspace consisting of immersions $\gamma$ passing through the base point $*$ and has $v \in T_{*} M$ as the unit tangent vector at $t=0$, i.e., $\gamma(0)=*$ and $\gamma^{\prime}(0) /\left\|\gamma^{\prime}(0)\right\|=v$. The map $D$ is actually a fibre bundle map:

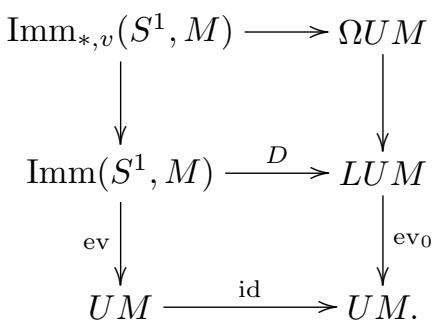

Here $\operatorname{ev}(\gamma)=\left(\gamma(0), \gamma^{\prime}(0) /\left\|\gamma^{\prime}(0)\right\|\right)$, and $\Omega U M$ is the based loop spaces of $U M$ with $(*, v)$ as the base point. Since $D$ is a homotopy equivalence between the total spaces and the map between the base spaces is the identity map, we have:

Corollary 2.3. For a smooth manifold $M$, the 1-jet map

$$
D: \operatorname{Imm}_{*, v}\left(S^{1}, M\right) \rightarrow \Omega U M
$$

is a homotopy equivalence.

Since the space of long immersions plays an important role in this paper, let us explain how we can determine the homotopy type of $\operatorname{Imm}^{l}\left(\mathbb{R}, \mathbb{R}^{n}\right)$ : Again by the Hirsch-Smale theorem, the 1-jet map $D: \operatorname{Imm}\left(I, B^{n}\right) \rightarrow P U B^{n}$ is a homotopy equivalence. Here $P U B^{n}$ is the path space of the unit sphere bundle of the unit disc $B^{n}$. Consider the pull back diagram

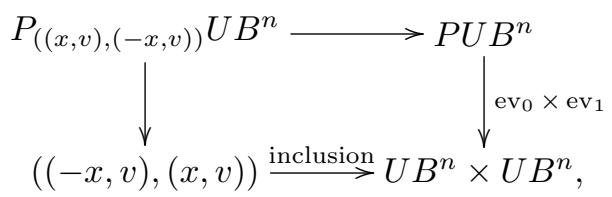


where $x=(1,0, \ldots, 0) \in B^{n}, v=(1,0, \ldots, 0) \in S^{n-1}=T_{x} B^{n}$ and $\mathrm{ev}_{i}$ maps a path $\gamma$ to $\gamma(i), i=0,1$. This diagram essentially defines the space of path $P_{(-x, v),(x, v)} U B^{n}$ between $(x, v)$ and $(-x, v)$ in $U B^{n}$. On the other hand, $D$ is a bundle map with $U B^{n}$ as the base:
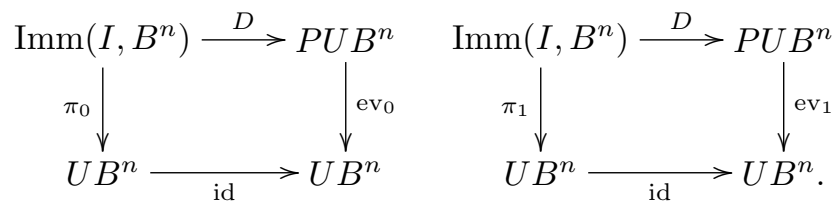

Here $\pi_{i}(\gamma)=\left(\gamma(i), \gamma^{\prime}(i) /\left\|\gamma^{\prime}(i)\right\|\right)$ for $\gamma \in \operatorname{Imm}\left(I, B^{n}\right)$ and $i=0,1$. Therefore we conclude that $D:\left(\pi_{0} \times \pi_{1}\right)^{-1}((-x, v)(x, v)) \rightarrow P_{(-x, v),(x, v))} U B^{n}$ is a homotopy equivalence, where $\left(\pi_{0} \times \pi_{1}\right)^{-1}((-x, v),(x, v))$ is defined by the pull back diagram

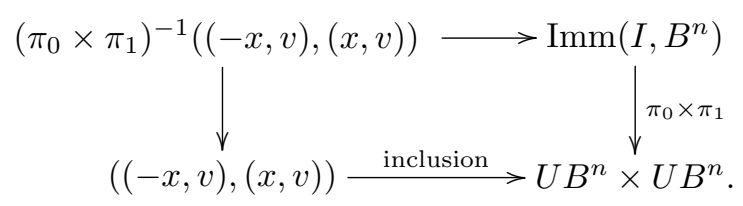

On the other hand, $\left(\pi_{0} \times \pi_{1}\right)^{-1}((-x, v),(x, v)) \subset \operatorname{Imm}\left(I, B^{n}\right)$ is essentially the space of long immersions $\operatorname{Imm}^{l}\left(\mathbb{R}, \mathbb{R}^{n}\right)$, and $P_{((-x, v),(x, v))} U B^{n}$ is homeomorphic to $P_{(-x, x)} B^{n} \times \Omega_{v} S^{n-1}$ because the tangent bundle of $B^{n}$ is trivializable. It is clear that $P_{((-x, v),(x, v))} B^{n}$ is contractible; therefore, $\operatorname{Imm}^{l}\left(\mathbb{R}, \mathbb{R}^{n}\right)$ is homotopy equivalent to $\Omega S^{n-1}$.

Corollary 2.4. For all $n$, the composite map $\pi \circ D: \operatorname{Imm}^{l}\left(\mathbb{R}, \mathbb{R}^{n}\right) \rightarrow \Omega S^{n-1}$ is a homotopy equivalence. Here $\pi: P U B^{n} \rightarrow P S^{n-1}$ is the projection on the second factor via the identification $P U B^{n} \sim P B^{n} \times P S^{n-1}$.

\section{Stringy operations}

In this section we recall the general framework where some of the string topology operations can be defined. This was observed by several authors independently, including the authors of this article. However, the main published reference is GruherSalvatore's paper [GS] which we follow closely. Let $F \rightarrow E \stackrel{\pi}{\rightarrow} M$ be a fibre bundle over a closed compact manifold $M$ of dimension $d$. We suppose that $E$ is equipped with a fibrewise associative product, that is a fibre bundle map $m: E \times_{M} E \rightarrow E$ such that

$$
m(x, m(y, z))=m(m(x, y), z)
$$

Here $E \times{ }_{M} E$ is defined via the pull back diagram

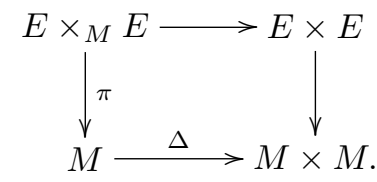




\section{The product}

Let us briefly explain how, following the main idea of $[\mathbf{C J}]$, this setup provides us with an associative product on $H_{*+d}(E)$, the homology of $E$ with shifted degree. Note that the natural map $E \times_{M} E \rightarrow E \times E$ is an embedding equipped with a tubular neighborhood obtained by pulling back $\nu$, the tubular neighborhood of the diagonal $M \stackrel{\Delta}{\rightarrow} M \times M$. The product $\mu: H_{i}(E) \otimes H_{j}(E) \rightarrow H_{i+j-d}(E)$ is defined by composing (from left to right) the maps:

(1) the Eilenberg-Zilber map $H_{*}(E) \otimes H_{*}(E) \rightarrow H_{*}(E \times E)$,

(2) the map $H_{*}(E \times E) \rightarrow H_{*}\left(\operatorname{Th}\left(\pi^{*}(\nu)\right)\right.$ induced by the Thom-Pontryagin collapse $\operatorname{map} E \times E \rightarrow\left(\operatorname{Th}\left(\pi^{*}(\nu)\right)\right.$,

(3) the Thom isomorphism $H_{*}\left(\operatorname{Th}\left(\pi^{*}(\nu)\right) \rightarrow H_{*-d}\left(E \times_{B} E\right)\right.$,

(4) and then the map induced by $m$ on homology $m_{*}: H_{*-d}\left(E \times_{B} E\right) \rightarrow H_{*}(E)$.

The associativity of this product, which follows from the associativity of $m$ and the associativity of multiple diagonal maps, is standard (for more details see [CJ] or $[\mathbf{G S}])$.

\section{The functoriality of the product}

The product defined above is natural in the following sense: Suppose

$$
\pi_{i}:\left(E_{i}, m_{i}\right) \rightarrow M
$$

are two fibrewise monoids and $h: E_{1} \rightarrow E_{2}$ is a map of fibrations over $M$. We have an induced map $h_{\Delta}: E_{1} \times_{M} E_{1} \rightarrow E_{2} \times_{M} E_{2}$. Then $h$ is said to be a map of fibrewise monoids if $m_{2}\left(h_{\Delta}\left(e_{1}, e_{2}\right)\right)=h\left(m_{1}\left(e_{1}, e_{2}\right)\right)$.

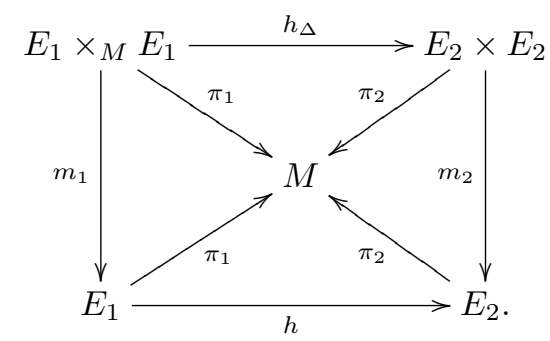

If $h:\left(E_{1}, m_{1}\right) \rightarrow\left(E_{2}, m_{2}\right)$ is a map of fibrewise monoids, then

$$
h_{*}:\left(H_{*}\left(E_{1}\right), \mu_{1}\right) \rightarrow\left(H_{*}\left(E_{2}\right), \mu_{2}\right)
$$

is a map of algebras. Since $\pi_{2} h=\pi_{1}, h$ induces a bundle map $h_{\mathrm{Th}}: \pi_{1}^{*}(\operatorname{Th}(v)) \rightarrow$ $\pi_{2}^{*}(\operatorname{Th}(v))$,

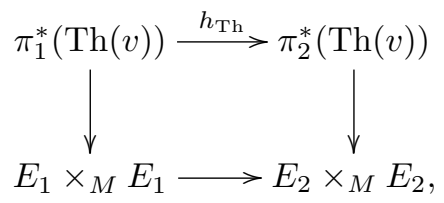


which makes the following diagram commute:

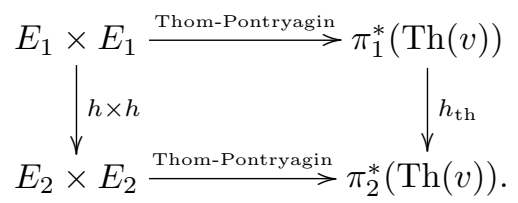

Now the naturality of the Thom isomorphism and the Eilenberg-Zilber map imply the claim.

\section{Compatibility with $m_{*}$ on $H_{*}(F)$}

Here we give a quick description of the wrong-way map $i$ ! : $H_{*}(E) \rightarrow H_{*-d}(F)$ associated to the inclusion $i: F \hookrightarrow E$. More details can be found in [GS]. One has the pull back diagrams
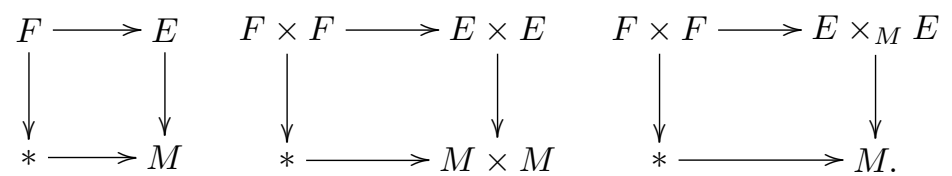

The wrong-way map for the left diagram is given by composing the map induced by the Thom-Pontryagin collapse map $H_{*}(E) \rightarrow H_{*}(\mathrm{Th}(F))$ and the Thom isomorphism $H_{*}(\operatorname{Th}(F)) \rightarrow H_{*-d}(F)$. The wrong-way map for the middle and right diagrams are constructed in a similar fashion.

For example, if the normal bundle of $F$ in $E$ is trivial, the Thom space of $F \hookrightarrow E$ is the suspension space $\Sigma^{d} F$. Then the wrong-way (shriek) map is given by composing the Thom-Pontryagin collapse map $H_{*}(E) \rightarrow H_{*}\left(\Sigma^{d} F\right)$ and the (special case of) Thom isomorphism $H *\left(\Sigma^{d} F\right) \rightarrow H_{*-d}(F)$.

Similarly, the Thom spaces of $F \times F \hookrightarrow E \times E$ and $F \times F \hookrightarrow E \times{ }_{M} E$ respectively are $\Sigma^{d}(F \times F)$ and $\Sigma^{2 d}(F \times F)$, and one gets the shriek maps $i !_{\Delta}: H_{*}\left(E \times{ }_{M} E\right) \rightarrow$ $H_{*-d}(F \times F)$ and $(i \times i) !: H_{*}(E \times E) \rightarrow H_{*-2 d}(F \times F)$ :

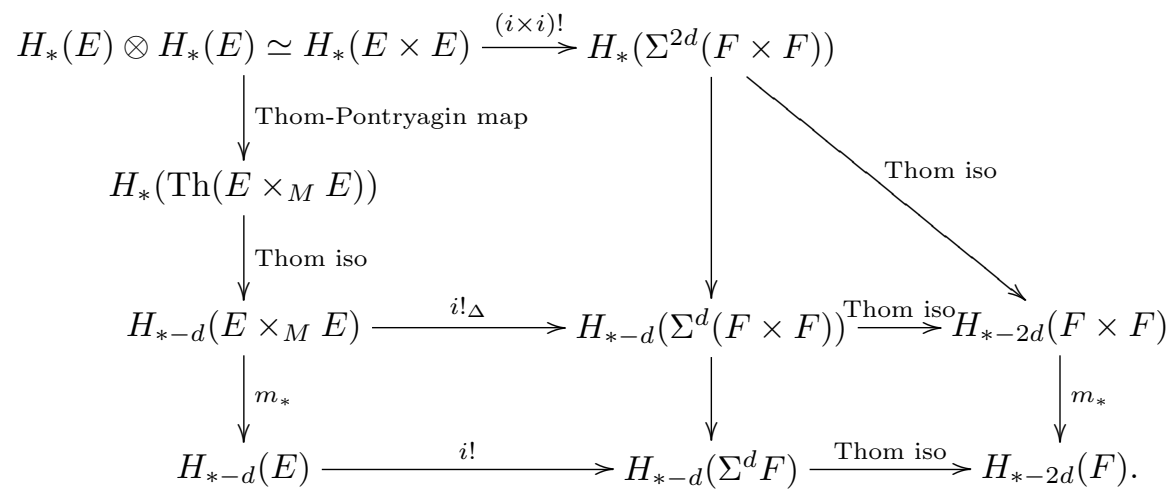

Let us summarize the discussion above:

Proposition 3.1. Let $\left(E_{i}, m_{i}\right) \rightarrow M, i=1,2$ be two fibrewise monoids over a closed oriented manifold $M$ of dimension d. Let $\mathbb{H}_{*}\left(E_{i}\right):=H_{*+d}\left(E_{i}\right)$ be the homology with a 
shift in degree by $-d$. Then $\mathbb{H}_{*}\left(E_{i}\right)$ can be naturally equipped with a graded associative algebra structure such that:

(1) The map $\left(\pi_{i}\right)_{*}: \mathbb{H}_{*}\left(E_{i}\right) \rightarrow \mathbb{H}_{*}(M)$ induced by the fibration map $\pi_{i}$ is a map of algebras. Here $\mathbb{H}_{*}(M)$ is equipped with the intersection production.

(2) The wrong-way map $\left(\mathbb{H}_{*}\left(E_{i}\right)=H_{*+d}\left(E_{i}\right), \mu\right) \rightarrow\left(H_{*}(F), m_{*}\right)$ induced by the inclusion of a fibre $F \hookrightarrow E$ is a map of algebras.

Moreover, if $h:\left(E_{1}, m_{1}\right) \rightarrow\left(E_{2}, m_{2}\right)$ is a morphism of fibrewise monoids over $M$, then the induced map $h_{*}: \mathbb{H}_{*}\left(E_{1}\right) \rightarrow \mathbb{H}_{*}\left(E_{2}\right)$ is a map of graded associative algebras.

Example 3.2. Consider $L M=C^{0}\left(S^{1}, M\right)$ the free loop space of a closed oriented manifold of dimension $d$. One has the fibration $\mathrm{ev}_{0}: L M \rightarrow M$, where $\mathrm{ev}_{0}$ associates to a loop $\gamma: S^{1} \rightarrow M$ its marked point $\gamma(0) \in M$. A typical fibre of this fibration is the based loop space $\Omega M$ whose concatenation product is not strictly associative but only up to homotopy. However this is not a major obstacle since one can give another model for this fibration, where the fibre has a strictly associative product. Note that $C^{\infty}\left(S^{1}, M\right)$ is homotopy equivalent to the set of continuous maps

$$
L M:=\{\gamma:[-a, a] \rightarrow M \mid a>0, \gamma(-a)=\gamma(a)\} .
$$

Now one can consider the fibration $\mathrm{ev}_{0}: L M \rightarrow M, \operatorname{ev}_{0}(\gamma)=\gamma(a)$, whose fibre is the Moore loop space

$$
\Omega M:=\{\gamma:[-a, a] \rightarrow M \mid a, \gamma(a)=\gamma(-a)=*\},
$$

whose concatenation product is

$$
\gamma_{1} * \gamma_{2}(t)= \begin{cases}\gamma_{1}\left(t+a_{2}\right) & \text { if }-a_{1}-a_{2} \leqslant t \leqslant a_{1}-a_{2} \\ \gamma_{2}\left(t-a_{1}\right) & \text { if } a_{1}-a_{2} \leqslant t \leqslant a_{1}+a_{2} .\end{cases}
$$

This model gives rise to the Chas-Sullivan product

$$
\text { • : } H_{i}(L M) \otimes H_{j}(L M) \rightarrow H_{i+j-d}(L M) .
$$

After regarding $\mathbb{H}_{i}(L M)=H_{i+d}(L M)$, one obtains a graded associative algebra $(\mathbb{H}(L M), \bullet)$. Moreover, the induced map ev $0: \mathbb{H}_{*}(L M) \rightarrow \mathbb{H}_{*}(M)$ is a map of associative algebras. It turns out that $\bullet$ is in fact commutative, but this is proved by using an argument that cannot be generalized to all fibrewise monoids.

Since in most of the interesting cases the fibrewise product $m$ is not (strictly) associative, one must adapt this definition so that it accommodates homotopy associative products and consider fibrewise homotopy monoids. This is better done by acquiring an operadic approach. One considers the trivial bundles $\overline{\mathcal{C}}_{n}=\mathcal{C}_{n} \times M \rightarrow M$, where $\left\{\mathcal{C}_{n}\right\}$ is an operad (see [GS, Section 5]). They form an operad $\overline{\mathcal{C}}$ in the category of fibred spaces over $M$. The existence of a $\overline{\mathcal{C}}$-algebra structure on a fibre bundle $E \stackrel{\pi}{\rightarrow} M$ is equivalent to having a collection of fibre bundle maps

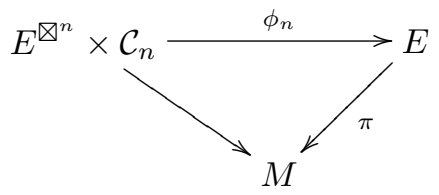

subject to the usual axioms for the algebras over an operad. Here $E^{\bigotimes^{n}}$ is defined by 
the pull back diagram

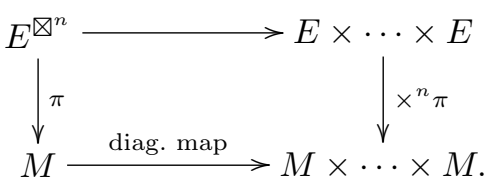

In particular, one can obtain a tubular neighborhood for the embedding $E^{\otimes^{n}} \hookrightarrow$ $E \times \cdots \times E$ by pulling back the total space of a normal bundle $\nu$ of the embedding $M \stackrel{\text { diagonal }}{\longrightarrow} M \times \cdots \times M$ by $\pi$.

The operadic operation $\mu_{n}: H_{*}\left(\mathcal{C}_{n}\right) \otimes H_{*}(E)^{\otimes n} \rightarrow H_{*}(E)$ is defined by composing:

(1) the tensor product of $\operatorname{id}_{H_{*}\left(\mathcal{C}_{n}\right)}$ and the Eilenberg-Zilber map: $H_{*}\left(\mathcal{C}_{n}\right) \otimes H_{*}(E)^{\otimes n}$ $\rightarrow H_{*}\left(\mathcal{C}_{n}\right) \otimes H_{*}(E \times \cdots \times E)$,

(2) the tensor product of $\operatorname{id}_{H_{*}\left(\mathcal{C}_{n}\right)}$ and the map induced by the Thom-Pontryagin collapse map: $H_{*}\left(\mathcal{C}_{n}\right) \otimes H_{*}(E \times \cdots \times E) \rightarrow H_{*}\left(\mathcal{C}_{n}\right) \otimes H_{*}\left(\operatorname{Th}\left(\pi^{*}(\nu)\right)\right.$,

(3) the tensor product of $\operatorname{id}_{H_{*}\left(\mathcal{C}_{n}\right)}$ and the Thom isomorphism:

$$
H_{*}\left(\mathcal{C}_{n}\right) \otimes H_{*}\left(\operatorname{Th}\left(\pi^{*}(\nu)\right) \rightarrow H_{*}\left(\mathcal{C}_{n}\right) \otimes H_{*-(n-1) d}\left(E^{\bigotimes^{n}}\right),\right.
$$

(4) the Eilenberg-Zilber map: $H_{*}\left(\mathcal{C}_{n}\right) \otimes H_{*-d}\left(E^{\bigotimes^{n}}\right) \rightarrow H_{*}\left(\mathcal{C}_{n} \times E^{\bigotimes^{n}}\right)$,

(5) the map induced by the operadic map $\phi, H_{*}\left(\mathcal{C}_{n} \times E^{\bigotimes^{n}}\right) \stackrel{\left(\phi_{n}\right)_{*}}{\longrightarrow} H_{*}(E)$.

Note that the map $\mu_{n}$ is of homological dimension $-(n-1) d$ since the Thom isomorphism corresponds to the integration along $(n-1) d$-dimensional fibre of the normal bundle. Therefore, to a homology class $x \in H_{k}\left(\mathcal{C}_{n}\right)$, one can associate a map

$$
H_{i_{1}}(E) \otimes H_{i_{2}}(E) \otimes \cdots \otimes H_{i_{n}}(E) \rightarrow H_{i_{1}+i_{2} \cdots i_{n}-(n-1) d+k}(E) .
$$

For instance, in the case of the little 2-disk operad, a fibrewise action gives rise to a commutative and associative product of degree $-d$,

$$
\bullet: H_{*}(E) \times H_{*}(E) \rightarrow H_{*-d}(E),
$$

which corresponds to the action of the 0-dimensional class of $H_{*}\left(\mathcal{C}_{2}\right)$. There is also a Gerstenhaber bracket of degree $(1-d)$

$$
\{-,-\}: H_{*}(E) \times H_{*}(E) \rightarrow H_{*+1-d}(E),
$$

which satisfies the Jacobi identity for a Lie bracket of degree $1-d$. This bracket corresponds to the action of a generator of $H_{1}\left(\mathcal{C}_{2}\right)$, which can be described as follows: fix a disc at the center of the the unit disk and consider another disk with sufficiently small radius moving around the first one, such that its center always lays on a fixed circle whose center is the center of the unit disk. This represents a one-dimensional homology class in $H_{1}\left(\mathcal{C}_{2}\right)$.

Note that having an action of the little 2-cube operad is much stronger than having a homotopically associative product, and it also implies that the product is commutative up to homotopy. The combined works of Budney [B1, Corollary 10] and Salvatore [S, Main Theorem] imply that:

Theorem 3.3 (R. Budney and P. Salvatore). For all n, the space of long knots $\operatorname{Emb}^{l}\left(\mathbb{R}, \mathbb{R}^{n}\right)$ admits an action of the little 2 -cube operad. The underlying product on 
$\operatorname{Emb}^{l}\left(\mathbb{R}, \mathbb{R}^{n}\right)$ is the usual concatenation which is, in particular, homotopy associative and commutative.

Let $\mathbb{H}_{*}\left(\operatorname{Emb}\left(S^{1}, S^{n}\right)\right):=H_{*+2 n-1}\left(\operatorname{Emb}\left(S^{1}, S^{n}\right)\right)$ be the homology of $\operatorname{Emb}\left(S^{1}, S^{n}\right)$ after a shift in the degree by $-2 n+1$ and, similarly, $\mathbb{H}_{*}\left(U S^{n}\right):=H_{*+2 n-1}\left(U S^{n}\right)$.

Theorem 3.4. There is a commutative and associative algebra structure on $\mathbb{H}_{*}\left(\operatorname{Emb}\left(S^{1}, S^{n}\right)\right)$, which makes $\mathrm{ev}_{*}: \mathbb{H}_{*}\left(\operatorname{Emb}\left(S^{1}, S^{n}\right)\right) \rightarrow \mathbb{H}_{*}\left(U S^{n}\right)$, the map induced by projection on the unit tangent vector, into an algebra map. Here $\mathbb{H}_{*}\left(U S^{n}\right)$ is equipped with the standard intersection product.

Proof. Budney and Cohen ([BC, Proposition 4.3]) had observed that $\operatorname{Emb}\left(S^{1}, S^{n}\right)$ is homotopy equivalent to $S O(n+1) \times_{S O(n-1)} \operatorname{Emb}^{l}\left(\mathbb{R}, \mathbb{R}^{n}\right)$. The latter is the total space of the standard fibration

$$
\pi: S O(n+1) \times_{S O(n-1)} \operatorname{Emb}^{l}\left(\mathbb{R}, \mathbb{R}^{n}\right) \rightarrow S O(n+1) / S O(n-1),
$$

which is equipped with a fibrewise action of the little disk operad as follows: By the Budney-Salvatore theorem, one has an action of the little disk operad

$$
\phi_{k}^{1}: \mathcal{C}_{k} \times\left(\operatorname{Emb}^{l}\left(\mathbb{R}, \mathbb{R}^{n}\right)\right)^{\times k} \rightarrow \operatorname{Emb}^{l}\left(\mathbb{R}, \mathbb{R}^{n}\right) .
$$

The key point is that this operadic action is $S O(n-1)$-equivariant. This property is transparent in Budney's construction [B1], and for Salvatore's double loop space structure, we refer the reader to the proof of Theorem 1.4 in [S, page 19]. A direct computation shows that

$$
\begin{aligned}
\left(S O(n+1) \times_{S O(n-1)}\right. & \left.\operatorname{Emb}^{l}\left(\mathbb{R}, \mathbb{R}^{n}\right)\right)^{\bigotimes^{k}} \\
& =S O(n+1) \times_{S O(n-1)}(\underbrace{\operatorname{Emb}^{l}\left(\mathbb{R}, \mathbb{R}^{n}\right) \times \cdots \operatorname{Emb}^{l}\left(\mathbb{R}, \mathbb{R}^{n}\right)}_{k-\text { times }}) .
\end{aligned}
$$

Here the action of $S O(n-1)$ on $\operatorname{Emb}^{l}\left(\mathbb{R}, \mathbb{R}^{n}\right) \times \cdots \operatorname{Emb}^{l}\left(\mathbb{R}, \mathbb{R}^{n}\right)$ is diagonal. We define the fibrewise operadic actions

$$
\begin{aligned}
& \phi_{k}: \mathcal{C}_{k} \times\left[S O(n+1) \times_{S O(n-1)}(\underbrace{\operatorname{Emb}^{l}\left(\mathbb{R}, \mathbb{R}^{n}\right) \times \cdots \times \operatorname{Emb}^{l}\left(\mathbb{R}, \mathbb{R}^{n}\right)}_{k-\text { times }})\right] \\
& \rightarrow S O(n+1) \times_{S O(n-1)} \operatorname{Emb}^{l}\left(\mathbb{R}, \mathbb{R}^{n}\right)
\end{aligned}
$$

to be $\phi_{k}\left(c,\left[a,\left(x_{1}, \ldots, x_{n}\right)\right]\right)=\left[a, \phi_{k}^{1}\left(c, x_{1}, \ldots, x_{n}\right)\right]$, where

$$
a \in S O(n+1), x_{i} \in \operatorname{Emb}^{l}\left(\mathbb{R}, \mathbb{R}^{n}\right), c \in \mathcal{C}_{n} .
$$

Since $\phi_{k}^{1}$ 's are $S O(n-1)$-equivariant, $\phi_{k}$ 's are well-defined and satisfy the axioms of an operadic action. One immediately obtains a commutative and associative product of degree $-2 n+1=-\operatorname{dim}(S O(n+1) / S O(n-1))$ :

$$
\mu_{\mathrm{em}}(-,-)=H_{*}\left(\operatorname{Emb}\left(S^{1}, S^{n}\right)\right) \times H_{*}\left(\operatorname{Emb}\left(S^{1}, S^{n}\right)\right) \rightarrow H_{*-2 n+1}\left(\operatorname{Emb}\left(S^{1}, S^{n}\right)\right),
$$

which after a shift in the degrees by $-2 n+1$ reads

$$
\mu_{\mathrm{em}}(-,-)=\mathbb{H}_{*}\left(\operatorname{Emb}\left(S^{1}, S^{n}\right)\right) \times \mathbb{H}_{*}\left(\operatorname{Emb}\left(S^{1}, S^{n}\right)\right) \rightarrow \mathbb{H}_{*}\left(\operatorname{Emb}\left(S^{1}, S^{n}\right)\right) .
$$

Now we prove the second part of the statement. Consider the projection

$$
\text { ev: } \operatorname{Emb}\left(S^{1}, S^{n}\right) \rightarrow H_{*}\left(U S^{n}\right), \operatorname{ev}(\gamma)=\left(\gamma(0), \gamma^{\prime}(0) /\left\|\gamma^{\prime}(0)\right\|\right) .
$$


By the commutative diagram (2.1), ev is identified with the projection on the first factor $\pi: S O(n+1) \times_{S O(n-1)} \mathrm{Emb}^{l}\left(\mathbb{R}, \mathbb{R}^{n}\right)$. Note that by the pull back diagram

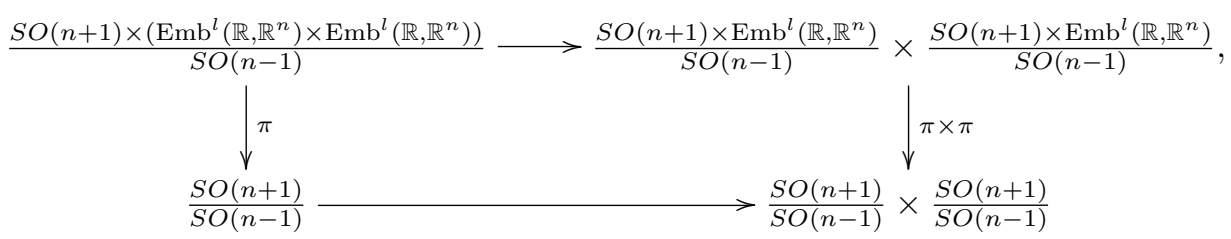

the normal bundle of embedding

$$
\frac{S O(n+1) \times\left(\mathrm{Emb}^{l}\left(\mathbb{R}, \mathbb{R}^{n}\right) \times \mathrm{Emb}^{l}\left(\mathbb{R}, \mathbb{R}^{n}\right)\right)}{S O(n-1)} \longrightarrow \frac{S O(n+1) \times \mathrm{Emb}^{l}\left(\mathbb{R}, \mathbb{R}^{n}\right)}{S O(n-1)} \times \frac{S O(n+1) \times \mathrm{Emb}^{l}\left(\mathbb{R}, \mathbb{R}^{n}\right)}{S O(n-1)}
$$

is the pull back of the normal bundle of the diagonal embedding

$$
\frac{S O(n+1)}{S O(n-1)} \rightarrow \frac{S O(n+1)}{S O(n-1)} \times \frac{S O(n+1)}{S O(n-1)}
$$

by $\pi$. Therefore we have the commutative diagram

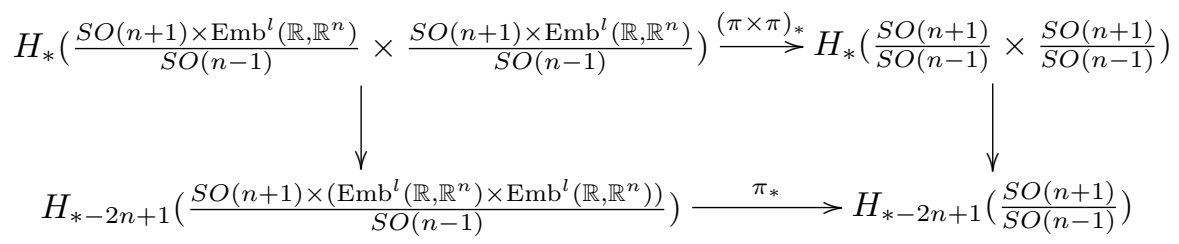

where the vertical arrows are given by composing the Thom isomorphisms and the Thom-Pontryagin collapse maps. Next we have the commutative diagram

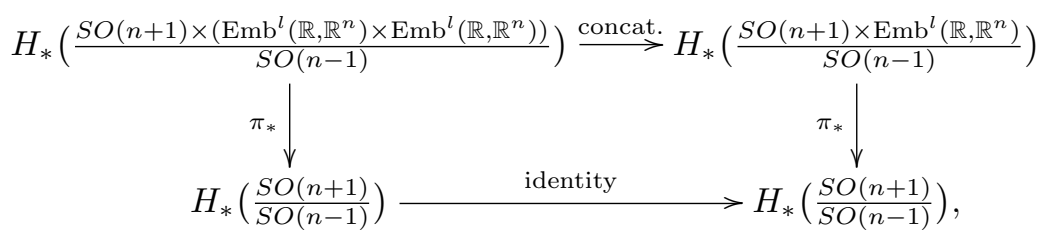

where the top horizontal arrow is given by the concatenation. By fitting together these two commutative diagrams and the naturality of the Eilenberg-Zilberg isomorphism, one deduces that $\pi_{*}$ is a morphism of algebras. We recall that the map given by composing the Thom-Pontryagin collapse map and the Thom isomorphism,

$$
\begin{array}{r}
H_{*}\left(\frac{S O(n+1)}{S O(n-1)}\right) \otimes H_{*}\left(\frac{S O(n+1)}{S O(n-1)}\right) \\
\text { Eilenberg-Zilber } \mid \simeq \\
H_{*}\left(\frac{S O(n+1)}{S O(n-1)} \times \frac{S O(n+1)}{S O(n-1)}\right) \\
\text { Thom-Pontryagin. collapse + Thom iso. } \\
H_{*-2 n+1}\left(\frac{S O(n+1)}{S O(n-1)}\right),
\end{array}
$$

is precisely the intersection product that can be alternatively defined using the 
Poincaré duality for the compact manifold $\frac{S O(n+1)}{S O(n-1)}$.

As mentioned in the previous section, $\operatorname{Imm}^{\prime}\left(S^{1}, S^{n}\right)$ is homotopy equivalent to $S O(n+1) \times{ }_{S O(n-1)} \operatorname{Imm}^{l}\left(\mathbb{R}, \mathbb{R}^{n}\right)$, which is the total space of the fibration

$$
\operatorname{Imm}^{l}\left(\mathbb{R}, \mathbb{R}^{n}\right) \rightarrow S O(n+1) \times_{S O(n-1)} \operatorname{Imm}^{l}\left(\mathbb{R}, \mathbb{R}^{n}\right) \rightarrow S O(n+1) / S O(n-1) .
$$

The fibres have a homotopy associative product, but they do not accommodate an action of the little 2-disk operad. For instance, we lose the homotopy commutativity of the product. Since the fibrewise product is not strictly associative, one cannot apply the fibrewise monoid formulation neither but this is not a major obstacle. We only have to modify our model so that the fibre has a strictly associative product.

Let $\mathbb{H}_{*}\left(\operatorname{Imm}^{\prime}\left(S^{1}, S^{n}\right)\right):=H_{*+2 n-1}\left(\operatorname{Imm}^{\prime}\left(S^{1}, S^{n}\right)\right)$.

Theorem 3.5. There is a graded associative algebra structure on $\mathbb{H}_{*}\left(\operatorname{Imm}^{\prime}\left(S^{1}, S^{n}\right)\right)$ such that $\mathrm{ev}_{*}: \mathbb{H}_{*}\left(\operatorname{Imm}^{\prime}\left(S^{1}, S^{n}\right)\right) \rightarrow \mathbb{H}_{*}\left(U S^{n}\right)$, the map induced by projection on the unit tangent vector, is an algebra map. Here $\mathbb{H}_{*}\left(U S^{n}\right)$ is equipped with standard intersection product.

Proof. The space $\operatorname{Imm}^{l}\left(\mathbb{R}, \mathbb{R}^{n}\right)$ is homotopy equivalent to

$$
\begin{aligned}
& \operatorname{Imm}_{+}^{l}\left(\mathbb{R}, \mathbb{R}^{n}\right)=\left\{f: \mathbb{R} \rightarrow \mathbb{R}^{n} \text { immersion, } \operatorname{supp}(f) \subset(-a, a)\right. \text { and } \\
& \left.\qquad([-a, a]) \subset B^{n} \text { for some } a \in \mathbb{R}^{+}\right\} .
\end{aligned}
$$

In fact, $\operatorname{Imm}^{l}\left(\mathbb{R}, \mathbb{R}^{n}\right)$ is a deformation retract of $\operatorname{Imm}_{+}^{l}\left(\mathbb{R}, \mathbb{R}^{n}\right)$, and the retract $R$ is given by reparametrizing linearly $(-a, a)$ by $(-1,1)$. More precisely, for $f: \mathbb{R} \rightarrow \mathbb{R}^{n}$ with $\operatorname{supp}(f) \subset(-a, a)$, one writes $R(f)(t):=f(t a)$.

Note that the standard concatenation product given by $\operatorname{Imm}_{+}^{l}\left(\mathbb{R}, \mathbb{R}^{n}\right)$ is strictly associative. Therefore one can use $S O(n+1) \times_{S O(n-1)} \operatorname{Imm}_{+}^{l}\left(\mathbb{R}, \mathbb{R}^{n}\right)$ as a model for $\operatorname{Imm}^{\prime}\left(S^{1}, S^{n}\right)$, and one immediately obtains an associative product

$$
\mu_{\mathrm{im}}(-,-)=H_{*}\left(\operatorname{Imm}^{\prime}\left(S^{1}, S^{n}\right)\right) \otimes H_{*}\left(\operatorname{Imm}^{\prime}\left(S^{1}, S^{n}\right)\right) \rightarrow H_{*-2 n+1}\left(\operatorname{Imm}^{\prime}\left(S^{1}, S^{n}\right)\right) .
$$

The second part of the statement is proved just like in the previous theorem.

After restricting to immersions with $k$ and $l$ with transverse double points, the product is

$$
\begin{aligned}
& \mu_{\mathrm{im}}^{k, l}(-,-)=H_{*}\left(\operatorname{Imm}_{k}{ }_{k}\left(S^{1}, S^{n}\right)\right) \otimes H_{*}\left(\operatorname{Imm}_{l}{ }_{l}\left(S^{1}, S^{n}\right)\right) \\
& \rightarrow H_{*-2 n+1}\left(\operatorname{Imm}_{k+l}^{\prime}\left(S^{1}, S^{n}\right)\right) .
\end{aligned}
$$

Theorem 3.6. There is a collection of maps

$$
\mu_{\mathrm{im}}^{k, l}: H_{*}\left(\operatorname{Imm}_{k}{ }_{k}\left(S^{1}, S^{n}\right)\right) \times H_{*}\left(\operatorname{Imm}_{l}{ }_{l}\left(S^{1}, S^{n}\right)\right) \rightarrow H_{*-2 n+1}\left(\operatorname{Imm}_{k+l}^{\prime}\left(S^{1}, S^{n}\right)\right)
$$

such that

$$
\left.\mu_{\mathrm{im}}^{k+l, m}\left(\mu_{\mathrm{im}}^{k, l}(a, b), c\right)\right)=\mu_{\mathrm{im}}^{k, l+m}\left(a, \mu_{\mathrm{im}}^{l, m}(b, c)\right) .
$$

Stated slightly differently, $\left(\mathbb{H}_{*}\left(\mathrm{Emb}^{s}\left(S^{1}, S^{n}\right)\right), \mu^{s}\right)$ is an associative algebra, where

$$
\mathbb{H}_{*}\left(\mathrm{Emb}^{s}\left(S^{1}, S^{n}\right)\right)=\oplus_{k} H_{*+2 n-1}\left(\operatorname{Imm}_{k}^{\prime}\left(S^{1}, S^{n}\right)\right) \text { and } \mu^{s}=\oplus_{k, l} \mu_{\mathrm{im}}^{k, l} \text {. }
$$




\section{Comparison with the Chas-Sullivan product}

So far we have only defined an associative product on $\mathbb{H}_{*}\left(\operatorname{Imm}^{\prime}\left(S^{1}, S^{n}\right)\right)$ and $\mathbb{H}_{*}\left(\operatorname{Imm}_{k}{ }_{k}\left(S^{1}, S^{n}\right)\right)$ and not on $\mathbb{H}_{*}\left(\operatorname{Imm}\left(S^{1}, S^{n}\right)\right):=H_{*+2 n-1}\left(\operatorname{Imm}\left(S^{1}, S^{n}\right)\right)$. We first show how one can define an associative product on $\mathbb{H}_{*}\left(\operatorname{Imm}\left(S^{1}, S^{n}\right)\right)$, and then we will compare this product with those on $\mathbb{H}_{*}\left(\operatorname{Imm}^{\prime}\left(S^{1}, S^{n}\right)\right)$ and $\mathbb{H}_{*}\left(\operatorname{Imm}_{k}{ }_{k}\left(S^{1}, S^{n}\right)\right)$ via the maps induced by the inclusion $\operatorname{Imm}^{\prime}\left(S^{1}, S^{n}\right) \hookrightarrow \operatorname{Imm}\left(S^{1}, S^{n}\right)$.

Let $U S^{n}$ be the unit tangent bundle of the $n$-sphere $S^{n}$ and $L U S^{n}$ be its free loop spaces. We consider the 1-jet map $D: \operatorname{Imm}\left(S^{1}, S^{n}\right) \rightarrow L U S^{n}$

$$
D: \gamma \mapsto\left(\gamma, \gamma^{\prime} /\left\|\gamma^{\prime}\right\|\right) \text {. }
$$

By the Hirsch-Smale Theorem 2.2, $\mathbb{H}_{*}\left(\operatorname{Imm}\left(S^{1}, S^{n}\right)\right)$ is isomorphic to $\mathbb{H}_{*}\left(L U S^{n}\right)$, which, by Example 3.2, is equipped with the Chas-Sullivan product. Moreover, one has the commutative diagram

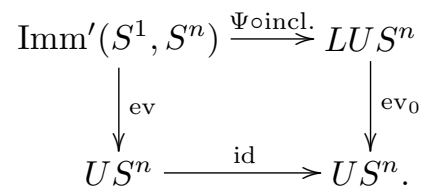

Here $\operatorname{ev}(\gamma)=\left(\gamma(0), \gamma^{\prime}(0) /\left\|\gamma^{\prime}(0)\right\|\right)$ for $\gamma \in \operatorname{Imm}^{\prime}\left(S^{1}, S^{n}\right)$, and $\operatorname{ev}_{0}(\alpha)=\alpha(0)$ for $\alpha \in$ $L U S^{n}$. As explained in the previous section, we can take a model for the fibration ev: $\operatorname{Imm}^{\prime}\left(S^{1}, S^{n}\right) \rightarrow U S^{n}$ so that the fibre is a strict monoid. It is clear that the two monoidal structures given in the proof of Theorem 3.5 and Example 3.2 are compatible, and the map

$$
D \circ \text { incl. } \circ \rho_{\mathrm{Imm}}: S O(n+1) \times_{S O(n-1)} \operatorname{Imm}^{l}\left(\mathbb{R}, \mathbb{R}^{n}\right) \simeq \operatorname{Imm}^{\prime}\left(S^{1}, S^{n}\right) \rightarrow L U S^{n}
$$

is a morphism of fibrewise monoids

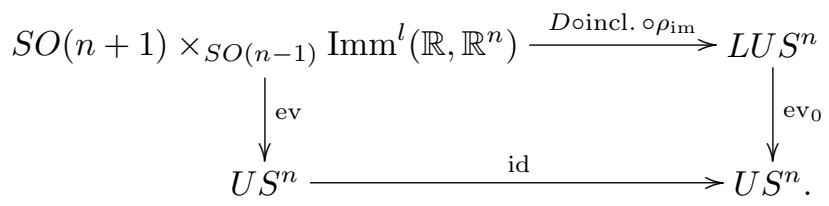

An immediate consequence of Proposition 3.1 is

Corollary 4.1. The map induced by the 1-jet map, $D_{*}:\left(\mathbb{H}_{*}\left(\operatorname{Imm}^{\prime}\left(S^{1}, S^{n}\right)\right), \mu_{\mathrm{im}}\right) \rightarrow$ $\left(\mathbb{H}_{*}\left(L U S^{n}\right), \bullet\right)$, is a map of graded associative algebras. Here, $\bullet$ is the Chas-Sullivan loop product.

\section{Desingularization morphism}

We first introduce the desingularization spaces $\mathfrak{Q}_{k}^{n}$ and the desingularization morphism $\sigma_{k}: \mathfrak{Q}_{k}^{n} \rightarrow \operatorname{Emb}\left(\mathbb{R}, \mathbb{R}^{n}\right)$. One should think of $\mathfrak{Q}_{k}^{n}$ as the space of $k$-singular long knots decorated with $k$ tangent vectors at the singular points, to be used to desingularize the knot (see $[\mathbf{C C L}])$.

Let $G_{2, n}=S O(n) /(S O(2) \times S O(n-2))$ be the Grassmanian manifold of oriented 2 -planes in $\mathbb{R}^{n}$. One has the canonical fibration $Q_{n} \rightarrow G_{2, n}$ whose fibres are $S^{n-3}$. 
Here $Q_{n}$ is the space of the pairs $(\pi, v)$, where $\pi$ is a 2-plane and $v$ is a vector perpendicular to $\pi$. More precisely,

$$
Q_{n}=S O(n) \times(S O(2) \times S O(n-2)) S^{n-3},
$$

where the action of $(S O(2) \times S O(n-2))$ on $S^{n-3}$ is trivial for the first factor and for the second factor is given by the natural action of $S O(n-2)$ on $S^{n-3} \subset \mathbb{R}^{n-2}$. Then $Q_{n}^{\times k}$ fibres over $G_{2, n}^{\times k}$ through the map $\left(\pi_{i}, v_{i}\right)_{1 \leqslant i \leqslant k} \mapsto\left(\pi_{i}\right)_{1 \leqslant i \leqslant k}$.

We define $\mathfrak{Q}_{n}^{k}$ to be the total space of the pull back of the fibration $Q_{n}^{\times k} \rightarrow G_{2, n}^{\times k}$ via the natural map $r_{k}: \operatorname{Imm}_{k}^{l}\left(\mathbb{R}, \mathbb{R}^{n}\right) \rightarrow G_{2, n}^{\times k}$, given by sending an immersion with $k$ singular double points to the $k 2$-planes spanned at the singularities by the tangent vectors. We have the following commutative diagram of fibrations:

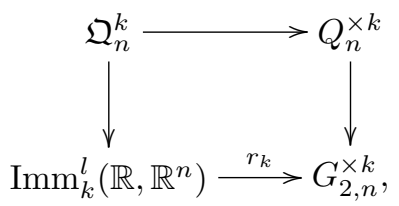

where the fibres are $\left(S^{n-3}\right)^{\times k}$.

The desingularization morphism $\sigma=\coprod \sigma_{k}: \coprod_{k \geqslant 1} \mathfrak{Q}_{n}^{k} \rightarrow \operatorname{Emb}^{l}\left(\mathbb{R}, \mathbb{R}^{n}\right)$ is defined by resolving the singularities using the normal vectors given at those points. More explicitly, let $\left(\gamma,\left(v_{i}\right)_{1 \leqslant i \leqslant k}\right) \in \mathfrak{Q}_{k}$, where $\gamma: \mathbb{R} \rightarrow \mathbb{R}^{n}$ is a long immersion with double singular points $\gamma\left(t_{1}^{1}\right)=\gamma\left(t_{2}^{1}\right), \gamma\left(t_{1}^{2}\right)=\gamma\left(t_{2}^{2}\right), \ldots, \gamma\left(t_{1}^{k}\right)=\gamma\left(t_{2}^{k}\right)$ and $v_{1}, \ldots, v_{k}$ are the vectors normal to the tangent plane at the singularities. We can take the $t_{j}^{i}$ 's to be pairwise ordered, i.e., $t_{1}^{i} \leqslant t_{2}^{i}$ for all $i$. Consider the bump functions

$$
\alpha_{i}(t)= \begin{cases}\delta \exp \left(\frac{-1}{\left(t-t_{1}^{i}\right)^{2}-\epsilon^{2}}\right) & \text { if } t \in\left[t_{1}^{i}-\epsilon, t_{1}^{i}+\epsilon\right] \\ -\delta \exp \left(\frac{-1}{\left(t-t_{2}^{i}\right)^{2}-\epsilon^{2}}\right) & \text { if } t \in\left[t_{2}^{i}-\epsilon, t_{2}^{i}+\epsilon\right] \\ 0 & \text { otherwise }\end{cases}
$$

where each $a_{i}$ is 1 or 2 . Let $\sigma\left(\left(\gamma,\left(v_{i}\right)_{i=1}^{k}\right)\right): \mathbb{R} \rightarrow \mathbb{R}^{n}$ be

$$
\sigma_{k}\left(\left(\gamma,\left(v_{i}\right)_{i=1}^{k}\right)\right)(t)=\gamma(t)+\sum_{i=1}^{k} \alpha_{i}(t) v_{i}
$$

For sufficiently small $\epsilon$ and $\delta, t \mapsto \sigma_{k}\left(\left(\gamma,\left(v_{i}\right)_{i=1}^{k}\right)\right)(t)$ is well-defined and is an embedding. So one considers the space $\mathfrak{Q}_{k}^{\prime n}$ consisting of the triples $\left[\left(\gamma,\left(v_{0} i\right)_{1 \leqslant i \leqslant k}\right), \epsilon, \delta\right]$, where $\epsilon, \delta$ are small enough so that the construction works, i.e., the outcome is an embedding. For each $\gamma$, the domain of admissible $\epsilon$ and $\delta$ continuously depends on $\gamma$. One has the fibration $\mathfrak{Q}_{k}^{\prime n} \rightarrow \mathfrak{Q}_{k}^{n}$, given by projecting on the first factor $\left(\gamma,\left(v_{i}\right)_{1 \leqslant i \leqslant k}\right)$, whose fibres are contractible. Therefore, $\mathfrak{Q}_{k}^{\prime n}$ and $\mathfrak{Q}_{k}^{n}$ have the same homotopy type. Then both $\mathfrak{Q}_{k}^{\prime n}$ and $\mathfrak{Q}_{k}^{n}$ are thought of as the space of long immersions decorated with some normal vectors at the singularities, and they have a natural homotopy associative product induced by concatenation.

Moreover, $\sigma_{k}$ is clearly $S O(n-1)$ equivariant; therefore it induces a homologically well-defined map

$$
\Sigma_{k}: H_{*}\left(S O(n+1) \times_{S O(n-1)} \mathfrak{Q}_{k}^{n}\right) \rightarrow H_{*}\left(S O(n+1) \times_{S O(n-1)} \operatorname{Emb}^{l}\left(\mathbb{R}, \mathbb{R}^{n}\right)\right) .
$$


We have a map

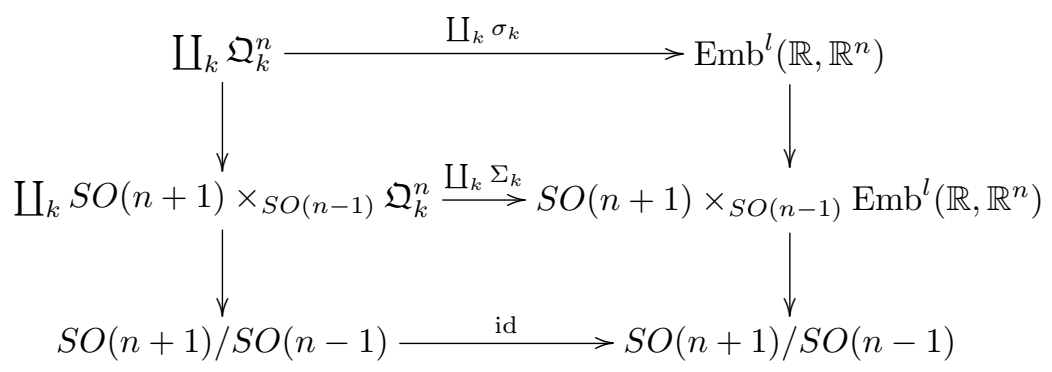

of fibrewise monoid. Hence

\section{Lemma 5.1.}

$$
\begin{aligned}
\oplus_{k} \Sigma_{k}:\left(\oplus _ { k } H _ { * } \left(S O(n+1) \times_{S O}(n-1)\right.\right. & \left.\left.\mathfrak{Q}_{k}^{n}\right), *\right) \\
& \rightarrow\left(H_{*}\left(S O(n+1) \times_{S O(n-1)} \operatorname{Emb}^{l}\left(\mathbb{R}, \mathbb{R}^{n}\right)\right), \mu_{\mathrm{im}}\right)
\end{aligned}
$$

is a map of associative algebras. Here the product $*$ is induced by the concatenation.

We need another map in order to complete the construction of the desingularization morphism from the homology of the space of immersions in $S^{n}$ to the homology of knot space. So far we have constructed a map from $H_{*}\left(\left(S O(n+1) \times_{S O(n-1)}\right.\right.$ $\left.\mathfrak{Q}_{k}^{n}\right)$ to $H_{*}\left(S O(n+1) \times_{S O(n-1)} \operatorname{Emb}^{l}\left(\mathbb{R}, \mathbb{R}^{n}\right)\right)$, and we need a map which takes from $H_{*}\left(\operatorname{Imm}_{k}\left(S^{1}, S^{n}\right)\right)$ to $H_{*}\left(\left(S O(n+1) \times_{S O(n-1)} \mathfrak{Q}_{k}^{n}\right)\right.$. The latter is given by the Gysin map associated to the projection map

$$
S O(n+1) \times_{S O(n-1)} \mathfrak{Q}_{k}^{n} \rightarrow\left(S O(n+1) \times_{S O(n-1)} \operatorname{Imm}_{k}^{l}\left(\mathbb{R}, \mathbb{R}^{n}\right)\right) \simeq \operatorname{Imm}_{k}\left(S^{1}, S^{n}\right) .
$$

The Gysin maps of fibrations have a convenient description using the spectral sequence, which makes its naturality property more transparent.

Proposition 5.2 (See $[\mathbf{B G}]$ ). Let $F \rightarrow E \rightarrow M$ be an oriented fibration whose fibre has the homotopy type of a closed manifold of dimension $p$. Then there is a natural map $f !: H_{*}(M) \rightarrow H_{*+p}(E)$. This is the dual of the map on the cohomology given by integration over fibres.

A description using spectral sequence is the following composite:

$$
E_{p, n}^{2}=H_{p}\left(M, \mathcal{H}_{n}(F)\right)=H_{p}(M, \mathbb{Z}) \hookrightarrow E_{p, n}^{\infty} \Rightarrow H_{p+n}(E) .
$$

Here $\mathcal{H}_{q}(F)$ stands for the local coefficient system, which is trivial in this case and has the fundamental class of $F$ as a generator (because the fibration is oriented). In this description one can clearly see the naturality property.

We apply this construction to the oriented fibration $\mathfrak{Q}_{k}^{n} \rightarrow \operatorname{Imm}_{k}^{l}\left(\mathbb{R}, \mathbb{R}^{n}\right)$ and $S O(n+1) \times_{S O(n-1)} \mathfrak{Q}_{k}^{n} \rightarrow S O(n+1) \times_{S O(n-1)} \operatorname{Imm}_{k}^{l}\left(\mathbb{R}, \mathbb{R}^{n}\right)$.

Therefore the fibration $\mathfrak{Q}_{k}^{n} \rightarrow \operatorname{Imm}_{k}^{l}\left(\mathbb{R}, \mathbb{R}^{n}\right)$ gives rise to the maps

$$
\theta_{k}: H_{*}\left(\operatorname{Imm}_{k}^{l}\left(\mathbb{R}, \mathbb{R}^{n}\right)\right) \rightarrow H_{*+k(n-3)}\left(\mathfrak{Q}_{k}^{n}\right)
$$

and

$$
\Theta_{k}: H_{*}\left(S O(n+1) \times_{S O(n-1)} \operatorname{Imm}_{k}^{l}\left(\mathbb{R}, \mathbb{R}^{n}\right)\right) \rightarrow H_{*+k(n-3)}\left(S O(n+1) \times_{S O(n-1)} \mathfrak{Q}_{k}^{n}\right) .
$$


Lemma 5.3. The Gysin maps $\Theta_{k}^{n}$ 's are multiplicative in the sense that

$$
\Theta_{k}(x) * \Theta_{l}(y)=\Theta_{k+l}\left(\mu_{\mathrm{im}}^{k, l}(x, y)\right) .
$$

Proof. If the fibration $F \rightarrow E \rightarrow M$ is a fibrewise monoid, then the Leray-Serre spectral sequence accommodates the fibrewise monoidal structure. In other words, all the pages, including $E^{\infty}$, of the spectral sequence have a multiplicative structure.

A reader interested in a more detailed proof is invited to see the Cohen-Jones-Yan paper $[\mathbf{C J Y}]$, where they give a detailed proof of the multiplicative structure of the Leray-Serre spectral sequence in the case of free loop space fibration. Their result and proof apply to our general case of fibrewise monoids.

We define the desingularization morphism to be the composite

$$
\begin{gathered}
\Sigma_{k} \circ \Theta_{k}: H_{*}\left(S O(n+1) \times_{S O(n-1)} \operatorname{Imm}_{k}^{l}\left(\mathbb{R}, \mathbb{R}^{n}\right)\right) \rightarrow \\
H_{*-k(n-3)}\left(S O(n+1) \times_{S O(n-1)} \operatorname{Emb}^{l}\left(\mathbb{R}, \mathbb{R}^{n}\right)\right), \\
\phi_{k}=\Sigma_{k} \circ \Theta_{k}: H_{*}\left(\operatorname{Imm}_{k}\left(S^{1}, S^{n}\right)\right) \rightarrow H_{*+k(n-3)}\left(\operatorname{Emb}\left(S^{1}, S^{n}\right)\right) .
\end{gathered}
$$

Theorem 5.4. The desingularization morphisms

$$
\phi_{k}: \mathbb{H}_{*}\left(\operatorname{Imm}_{k}{ }_{k}\left(S^{1}, S^{n}\right)\right) \rightarrow \mathbb{H}_{*}\left(\operatorname{Emb}\left(S^{1}, S^{n}\right)\right),
$$

$k \geqslant 0$, are compatible with the products, i.e., for $x \in \mathbb{H}_{*}\left(\operatorname{Imm}_{k}^{\prime}\left(S^{1}, S^{n}\right)\right)$ and $y \in$ $H_{*}\left(\operatorname{Imm}_{l}^{\prime}\left(\left(S^{1}, S^{n}\right)\right)\right)$

$$
\mu_{\mathrm{em}}\left(\phi_{k}(x), \phi_{l}(y)\right)=\phi_{k+l}\left(\mu_{\mathrm{im}}^{k, l}(x, y)\right) .
$$

Proof. For $x, y \in H_{*}\left(\operatorname{Imm}_{k}^{\prime}\left(S^{1}, S^{n}\right)\right)$,

$$
\mu_{\mathrm{em}}\left(\phi_{k}(x), \phi_{l}(y)\right)=\mu_{\mathrm{em}}\left(\Sigma_{k} \circ \Theta_{k}(x), \Sigma_{l} \circ \Theta_{l}^{n}(y)\right)=\Sigma_{k+l}\left(\Theta_{k}(x) * \Theta_{l}(y)\right)
$$

by Lemma 5.1. The latter is $\Sigma_{k+l} \circ \Theta_{k+l}\left(\mu_{\mathrm{im}}^{k, l}(x, y)\right)=\phi_{k+l}\left(\mu_{\mathrm{im}}^{k, l}(x, y)\right)$.

\section{Some computations}

Let us consider the canonical inclusion $i: \operatorname{Imm}^{\prime}\left(S^{1}, S^{n}\right) \rightarrow \operatorname{Imm}\left(S^{1}, S^{n}\right)$. The aim of this section is two-fold. First, we determine $i_{*}$, the morphism induced in singular homology $i_{*}$; secondly, we prove that the desingularization morphism is non-trivial.

Notation. For $V$ a graded $\mathbb{Z}$-module, $\Lambda(V)$ is the free exterior algebra generated by $V$, and $T(V)$ is the free tensor algebra generated by $V$.

\subsection{Homology of immersion space}

6.1.1. Immersions in $\mathbb{R}^{n+1}$.

As a warm-up example, we first consider the cases of long immersions $\operatorname{Imm}^{l}\left(\mathbb{R}, \mathbb{R}^{n+1}\right)$, and of singular knots $\operatorname{Imm}\left(S^{1}, \mathbb{R}^{n+1}\right)$ in an Euclidean space. Thanks to the HirschSmale theorem one knows that the 1-jet map, $\gamma \mapsto D \gamma$, is a homotopy equivalence 
from, respectively, $\operatorname{Imm}^{l}\left(\mathbb{R}, \mathbb{R}^{n+1}\right)$ and $\operatorname{Imm}\left(S^{1}, \mathbb{R}^{n+1}\right)$ to $P_{(-x, v),(x, v)} U \mathbb{R}^{n}$ (see Corollary 2.4) and $L U \mathbb{R}^{n+1}$, where $D(\gamma):=\left(\gamma, \frac{\dot{\gamma}}{\|\dot{\gamma}\|}\right)$.

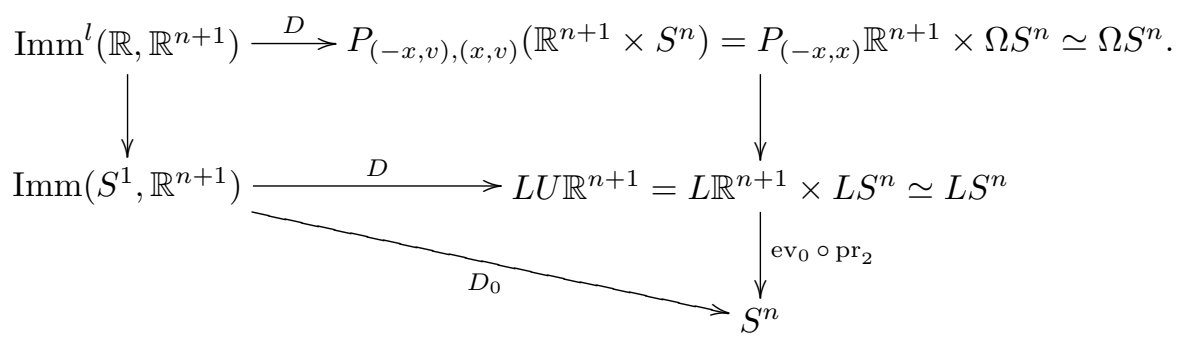

Note that one has the inclusion $\operatorname{Imm}^{l}\left(\mathbb{R}, \mathbb{R}^{n+1}\right) \hookrightarrow \operatorname{Imm}\left(S^{1}, \mathbb{R}^{n+1}\right)$, which corresponds to gluing the two end points of $\left.f\right|_{[-1,1]}$ for a given immersion $f \in \operatorname{Imm}^{l}\left(\mathbb{R}, \mathbb{R}^{n+1}\right)$ using a fixed path. After the identification $U \mathbb{R}^{n+1}$ together with $\mathbb{R}^{n+1} \times S^{n}$, the spaces $\operatorname{Imm}^{l}\left(\mathbb{R}, \mathbb{R}^{n+1}\right)$ and $\operatorname{Imm}\left(S^{1}, \mathbb{R}^{n+1}\right)$ are homotopy equivalent to $\Omega S^{n}$ and $L S^{n}$. The concatenation of long immersions in $\operatorname{Imm}^{l}\left(\mathbb{R}, \mathbb{R}^{n+1}\right)$ obviously corresponds to the composition of based loops in $\Omega S^{n}$. We recall that $H_{*}\left(\Omega S^{n}\right)$ with this multiplicative structure is isomorphic to the tensor algebra generated by an element $u$ of degree $n-1$. The evaluation map

$$
D_{0}: \operatorname{Imm}\left(S^{1}, \mathbb{R}^{n+1}\right) \stackrel{\mathrm{pr}_{2} \circ D}{\longrightarrow} L S^{n} \stackrel{\mathrm{ev}_{0}}{\longrightarrow} S^{n}
$$

can be used to endow the singular homology of $\operatorname{Imm}\left(S^{1}, \mathbb{R}^{n+1}\right)$ with a product. We consider the diagram

$$
\begin{gathered}
\operatorname{Imm}\left(S^{1}, \mathbb{R}^{n+1}\right) \times \operatorname{Imm}\left(S^{1}, \mathbb{R}^{n+1}\right)<^{j} \operatorname{Imm}\left(S^{1}, \mathbb{R}^{n+1}\right) \times{ }_{S^{n}} \operatorname{Imm}\left(S^{1}, \mathbb{R}^{n+1}\right) \\
\downarrow^{\operatorname{Tr}} \\
\operatorname{Imm}_{(0, \ldots, 0)}\left(\infty, \mathbb{R}^{n+1}\right) .
\end{gathered}
$$

Here $\operatorname{Imm}\left(S^{1}, \mathbb{R}^{n+1}\right) \times_{S^{n}} \operatorname{Imm}\left(S^{1}, \mathbb{R}^{n+1}\right)$ is the space of immersions $\left(\gamma_{1}, \gamma_{2}\right)$ such that $D_{0}\left(\gamma_{1}\right)=D_{0}\left(\gamma_{2}\right)$, and the space $\operatorname{Imm}_{(0, \ldots, 0)}\left(\infty, \mathbb{R}^{n+1}\right)$ is the subspace of $\operatorname{Imm}\left(S^{1}, \mathbb{R}^{n+1}\right) \times_{S^{n}} \operatorname{Imm}\left(S^{1}, \mathbb{R}^{n+1}\right)$ such that $\gamma_{1}(0)=\gamma_{2}(0)=(0, \ldots, 0)$. The map $\operatorname{Tr}$ is a translation map. It is defined using the unique translation $T_{1}$ that sends $\gamma_{1}(0)$ to $(0, \ldots, 0)$ and the unique translation $T_{2}$ that sends $\gamma_{2}(0)$ to $(0, \ldots, 0)$, $\operatorname{Tr}\left(\gamma_{1}, \gamma_{2}\right)=\left(T_{1}\left(\gamma_{1}\right), T_{2}\left(\gamma_{2}\right)\right)$. We also have a composition map

$$
\text { comp: } \operatorname{Imm}_{(0, \ldots, 0)}\left(\infty, \mathbb{R}^{n+1}\right) \rightarrow \operatorname{Imm}\left(S^{1}, \mathbb{R}^{n+1}\right) .
$$

As $j$ is a finite codimensional embedding, we can define a Gysin morphism $j$ ! and a product:

$$
-\bullet-:=\operatorname{comp}_{*} \circ \operatorname{Tr}_{*} \circ j_{!} \circ-\times-.
$$

This multiplicative structure is isomorphic to the Chas-Sullivan product on $H_{*+n}\left(L S^{n}\right)$, and the isomorphism is induced by the map $D$. The algebra $H_{*+n}\left(L S^{n}\right)$ has been computed by Cohen, Jones and Yan [CJY, Theorem 2]. For an oriented, 
closed manifold $M$ they consider the Serre spectral sequence associated to the evaluation fibration

$$
\Omega M \rightarrow L M \rightarrow M
$$

enriched with a multiplicative structure coming from the loop product of $\mathbb{H}_{*}(L M)$ lifted at the chain level. To be more precise, using the Pontryagin product on $H_{*}(\Omega M)$ and the intersection product on $\mathbb{H}_{*}(M)=H_{*+d}(M)$, the second page of this spectral sequence is ([CJY, Theorem 1])

$$
E_{p, q}^{2}:=\mathbb{H}_{p}\left(M, H_{q}(\Omega M)\right) \Rightarrow \mathbb{H}_{p+q}(L M):=H_{*+d}(L M) .
$$

For the spheres, they get

$$
\mathbb{H}_{*}\left(\operatorname{Imm}\left(S^{1}, \mathbb{R}^{n+1}\right)\right) \cong \mathbb{H}_{*}\left(L S^{n}\right) \cong \begin{cases}\Lambda(a) \otimes \mathbb{Z}[u] & \text { for } n \text { odd } \\ (\Lambda(b) \otimes \mathbb{Z}[a, v]) /\left(a^{2}, a b, 2 a v\right) & \text { for } n \text { even }\end{cases}
$$

with $a \in \mathbb{H}_{-n}\left(L S^{n}\right) \cong E_{-n, 0}^{\infty}, \quad b \in \mathbb{H}_{-1}\left(L S^{n}\right) \cong E_{-n, n-1}^{\infty}, \quad u \in \mathbb{H}_{n-1}\left(L S^{n}\right) \cong E_{0, n-1}^{\infty}$ and $v \in \mathbb{H}_{2 n-2}\left(L S^{n}\right) \cong E_{0,2 n-2}^{\infty}$.

6.1.2. Immersions in $S^{n}$.

The computation of $\mathbb{H}_{*}\left(\operatorname{Imm}\left(S^{1}, S^{n}\right)\right)$ has been completed by Chataur-Le Borgne in $[\mathbf{C L}]$. In fact Le Borgne in $[\mathbf{L}]$ considers the loop fibration

$$
L F \rightarrow L E \rightarrow L B
$$

associated to a smooth fibre bundle of oriented closed manifolds

$$
F \rightarrow E \rightarrow B
$$

Then he enriches the Serre spectral sequence with a multiplicative structure coming from the Chas-Sullivan loop product on $L B, L E$ and $L F$ :

$$
E_{p, q}^{2}:=\mathbb{H}_{p}\left(L B, \mathbb{H}_{q}(L F)\right) \rightarrow \mathbb{H}_{p+q}(L E) .
$$

In particular, he applies this new technique to the fibration

$$
L S^{n-1} \rightarrow L U S^{n} \rightarrow L S^{n},
$$

and he proves that the spectral sequence collapses at the $E^{2}$-term. For $n$ odd this is done directly and for $n$ even, using a comparison with the Serre spectral sequence of

$$
\Omega U S^{n} \rightarrow L U S^{n} \rightarrow U S^{n} .
$$

In both cases there are some serious extension issues which are all resolved in $[\mathbf{C L}$, Main Theorem] using Morse theoretical techniques: the authors filter the immersion spaces by the energy functional, and they get a new multiplicative spectral sequence that converges to $\mathbb{H}_{*}\left(\operatorname{Imm}\left(S^{1}, S^{n}\right)\right)$. We recall that we have the following isomorphisms of algebras:

Odd case. When $n$ is odd we have

$$
\mathbb{H}_{*}\left(\operatorname{Imm}\left(S^{1}, S^{n}\right)\right) \cong \mathbb{H}_{*}\left(L S^{n}\right) \otimes \mathbb{H}_{*}\left(L S^{n-1}\right) .
$$

Even case. When $n$ is even we have

$$
\mathbb{H}_{*}\left(\operatorname{Imm}\left(S^{1}, S^{n}\right)\right) \cong \mathbb{H}_{*}\left(U S^{n}\right) \otimes H_{*}\left(\Omega U S^{n}\right)
$$


We also recall:

\section{Lemma 6.1.}

$$
\mathbb{H}_{*}\left(U S^{n}\right) \cong \Lambda\left(a_{-n}, b_{-2 n+1}\right) /\left(2 a_{-n}, a_{-n} b_{-2 n+1}\right)
$$

and

$$
H_{*}\left(\Omega U S^{n}\right) \cong \mathbb{Z}\left[u_{n-2}, v_{2 n-2}\right] /\left(2 u_{n-2}\right) .
$$

Proof. The homology of $U S^{n}$ can be computed using the Serre spectral sequence of the fibration

$$
S^{n-1} \rightarrow U S^{n} \rightarrow S^{n} .
$$

As we want the multiplicative structure, we work in cohomology. We have

$$
E_{2}^{p, q}=H^{p}\left(S^{n}\right) \otimes H^{q}\left(S^{n-1}\right) \cong \Lambda\left(x_{n}\right) \otimes \Lambda\left(y_{n-1}\right) .
$$

The only non-trivial differential (for $n \geqslant 2$ ) is

$$
d_{n}: E_{n}^{0, n-1} \cong H^{n-1}\left(S^{n-1}\right) \rightarrow E_{n}^{n, 0} \cong H^{n}\left(S^{n}\right),
$$

which is the multiplication by 2 (the Euler characteristic), and we apply Poincaré duality to get the intersection product. The class $a_{-n}$ is Poincaré dual to the class of $x_{n}$ and $b_{-2 n+1}$ to the class of $x_{n} \otimes y_{n-1}$.

Similarly, $H_{*}\left(\Omega U S^{n}\right)$ can be computed using the Serre spectral sequence of

$$
\Omega S^{n-1} \rightarrow \Omega U S^{n} \rightarrow \Omega S^{n} .
$$

We get a multiplicative spectral sequence

$$
E_{p, q}^{2}=H_{p}\left(S^{n}\right) \otimes H_{q}\left(S^{n-1}\right) \cong T\left(w_{n-1}\right) \otimes T\left(u_{n-2}\right) .
$$

As it is a spectral sequence of algebra differentials are derivations, from the computation of the derivation

$$
d_{n-1}\left(w_{n-1}\right)=2 u_{n-2},
$$

we deduce the presentation of the Pontryagin algebra $H_{*}\left(\Omega S^{n}\right)$ given above where $v_{2 n-2}=u_{n-1}^{2}$. Let us explain why we have $d_{n-1}\left(w_{n-1}\right)=2 u_{n-2}$. As $U S^{n}$ is $n-2$ connected we know that

$$
H_{n-2}\left(\Omega U S^{n}\right) \cong H_{n-1}\left(U S^{n}\right) \cong \mathbb{Z} / 2 \mathbb{Z} .
$$

In order to produce this 2-torsion in the spectral sequence, we must have $d_{n-1}\left(w_{n-1}\right)$ $=2 u_{n-2}$.

In order to determine $i_{*}: \mathbb{H}_{*}\left(\operatorname{Imm}^{\prime}\left(S^{1}, S^{n}\right)\right) \rightarrow \mathbb{H}_{*}\left(\operatorname{Imm}\left(S^{1}, S^{n}\right)\right)$, we need to consider the fibrations

$$
\begin{aligned}
\operatorname{Imm}_{*}^{\prime}\left(S^{1}, S^{n}\right) & \rightarrow \operatorname{Imm}^{\prime}\left(S^{1}, S^{n}\right) \rightarrow S^{n} \\
\operatorname{Imm}_{*, v}^{\prime}\left(S^{1}, S^{n}\right) & \rightarrow \operatorname{Imm}^{\prime}\left(S^{1}, S^{n}\right) \rightarrow U S^{n},
\end{aligned}
$$

where $\mathrm{Imm}^{\prime}{ }_{*}$ denotes the space of based immersions and $\mathrm{Imm}^{\prime}{ }_{* v}$ is its subspace consisting of those immersions with a given unit tangent vector at the base point.

We notice that the second fibration admits a section geod. This map sends the unit tangent vector $v \in U S^{n}$ to the unique great circle such that $\dot{\gamma}(0)=v$. 
Theorem 6.2. For $n$ even number then

$$
\mathbb{H}_{*}\left(\operatorname{Imm}^{\prime}\left(S^{1}, S^{n}\right)\right) \cong \mathbb{H}_{*}\left(U S^{n}, H_{*}\left(\Omega S^{n-1}\right)\right) \cong \mathbb{H}_{*}\left(U S^{n}\right) \otimes H_{*}\left(\Omega S^{n-1}\right)
$$

The morphism $i_{*}$ is induced by the canonical morphism

$$
\mathbb{H}_{*}\left(U S^{n}, H_{*}\left(\Omega S^{n-1}\right)\right) \rightarrow \mathbb{H}_{*}\left(U S^{n}, H_{*}\left(\Omega U S^{n}\right)\right) \cong \mathbb{H}_{*}\left(\operatorname{Imm}\left(S^{1}, S^{n}\right)\right),
$$

which is the identity on $\mathbb{H}_{*}\left(U S^{n}\right)$, and the morphism

$$
j_{*}: H_{*}\left(\Omega S^{n-1}\right) \rightarrow H_{*}\left(\Omega U S^{n}\right)
$$

given by the inclusion of a fibre $j: S^{n-1} \rightarrow U S^{n}$. For odd number $n$,

$$
\mathbb{H}_{*}\left(\operatorname{Imm}^{\prime}\left(S^{1}, S^{n}\right)\right) \cong \mathbb{H}_{*}\left(S^{n}\right) \otimes \mathbb{H}_{*}\left(L S^{n-1}\right) .
$$

In this case the morphism $i_{*}$ is the injection

$$
\mathbb{H}_{*}\left(S^{n}\right) \otimes \mathbb{H}_{*}\left(L S^{n-1}\right) \stackrel{c_{*} \otimes \mathrm{Id}}{\longrightarrow} \mathbb{H}_{*}\left(L S^{n}\right) \otimes \mathbb{H}_{*}\left(L S^{n-1}\right),
$$

where $c: S^{n} \hookrightarrow L S^{n}$ is the inclusion of the constant loops.

Proof of the even case. Let us consider the Serre spectral sequence associated to the fibration

$$
\operatorname{Imm}_{*, v}^{\prime}\left(S^{1}, S^{n}\right) \rightarrow \operatorname{Imm}^{\prime}\left(S^{1}, S^{n}\right) \rightarrow U S^{n}
$$

The $E^{2}$-term of this spectral sequence is given by

$$
E_{p, q}^{2}=\mathbb{H}_{p}\left(U S^{n}, H_{q}\left(\operatorname{Imm}_{*, v}^{\prime}\left(S^{1}, S^{n}\right)\right)\right)
$$

Because $H_{*}\left(\Omega S^{n}\right)$ is torsion free, we have the canonical isomorphism

$$
E_{p, q}^{2} \cong \mathbb{H}_{p}\left(U S^{n}\right) \otimes H_{q}\left(\Omega S^{n-1}\right) .
$$

Let us recall that in the even case we have

$$
\mathbb{H}_{*}\left(U S^{n}\right) \cong \Lambda\left(a_{-n}, b_{-2 n+1}\right) /\left(2 a_{-n}, a_{-n} b_{-2 n+1}\right)
$$

and

$$
H_{*}\left(\Omega S^{n-1}\right) \cong \mathbb{Z}\left[u_{n-2}\right]
$$

In order to compute the other terms, we use the fact that this spectral sequence is multiplicative. As the underlying fibration has a section, we have $d_{k}\left(a_{-n}\right)=d_{k}\left(b_{-2 n+1}\right)$ $=0$ for $k \geqslant 2$, and we also have $d_{k}\left(u_{n-2}\right)=0$ for degree reasons. We conclude that this spectral sequence collapses at the $E^{2}$-term. Also, for degree reasons, there are no extension issues in that spectral sequence. In fact the $E^{2}$-term is concentrated on three columns, and it is easy to check that for any element $x \in \bigoplus_{p, q} E_{p, q}^{2}$ of total degree $m$, there exists a unique couple $(p, q)$ such that $m=p+q$ and $x \in E_{p, q}^{2}$. Thus we have that

$$
\mathbb{H}_{*}\left(\operatorname{Imm}^{\prime}\left(S^{1}, S^{n}\right)\right) \cong \Lambda\left(a_{-n}, b_{-2 n+1}\right) /\left(2 a_{-n}, a_{-n} b_{-2 n+1}\right) \otimes \mathbb{Z}\left[u_{n-2}\right]
$$


Now to identify $i_{*}$, note that $i$ is in fact a map of fibration,

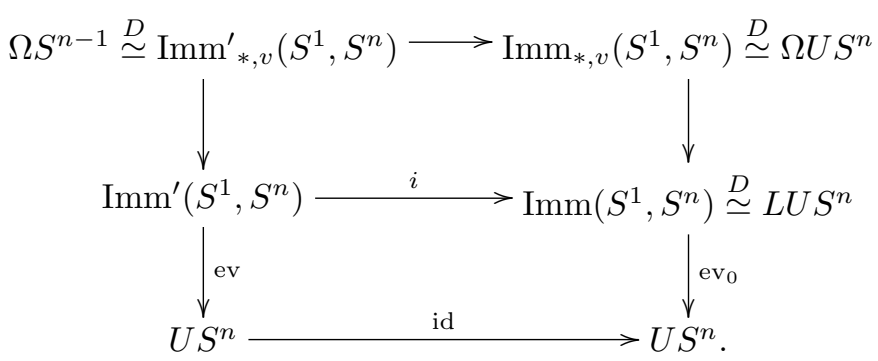

Therefore it induces a map between the $E_{2}$-pages of the Leray-Serre spectral sequences. Since the 1-jet map $D$ commutes with $i$ and both spectral sequences collapse at the $E_{2}$-term, the statement for the case $n$ even follows.

Proof of the odd case. In this case we consider the Leray-Serre spectral sequence associated to the fibration

$$
\operatorname{Imm}_{*}{ }_{*}\left(S^{1}, S^{n}\right) \rightarrow \operatorname{Imm}^{\prime}\left(S^{1}, S^{n}\right) \stackrel{\mathrm{ev}_{0}}{\longrightarrow} S^{n}
$$

because this fibration is comparable with the fibration $L S^{n-1} \rightarrow L U S^{n} \rightarrow L S^{n}$ whose Leray-Serre spectral sequence allows us to compute the homology of $\operatorname{Imm}\left(S^{1}, S^{n}\right) \stackrel{D}{\simeq}$ $L U S^{n}$. Stated more precisely, the 1-jet map is a map of fibrations:

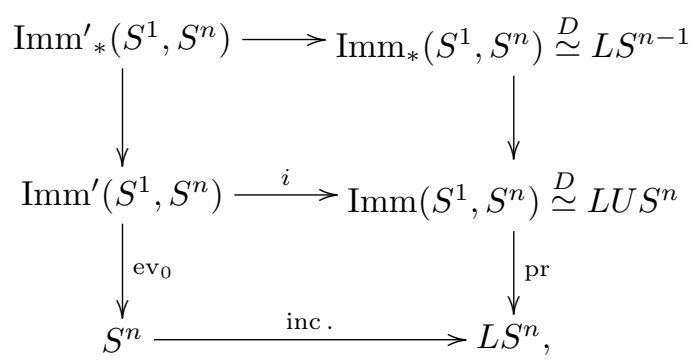

where inc.: $S^{n} \rightarrow L S^{n}$ is the inclusion of the constant loops in $L S^{n}$. On the other hand, the spectral sequence of the fibration (6.1) collapses at the $E_{2}$-term. To make this clear let us compute the homology of the fibre using the spectral sequence of the fibration

$$
\operatorname{Imm}_{*, v}^{\prime}\left(S^{1}, S^{n}\right) \rightarrow \operatorname{Imm}_{*}^{\prime}\left(S^{1}, S^{n}\right) \rightarrow S^{n-1} .
$$

We have that $H_{*}\left(\operatorname{Imm}_{*, v}^{\prime}\left(S^{1}, S^{n}\right)\right) \simeq H_{*}\left(\operatorname{Imm}^{l}\left(\mathbb{R}, \mathbb{R}^{n}\right) \simeq H_{*}\left(\Omega S^{n-1}\right) \simeq \mathbb{Z}\left[x_{n-2}\right]\right.$ is the polynomial algebra in one variable $x_{n-2}$ of degree $n-2$ and $\mathbb{H}_{*}\left(S^{n-1}\right) \simeq \Lambda y_{-n+1}$. So we have $E_{2}=\Lambda y_{-n+1} \otimes \mathbb{Z}\left[x_{n-2}\right]$. Clearly, $d_{2} x_{n-2}=0$ and $d_{2} y_{n-1}=0$ as this fibration has a section, and all elements of $\mathbb{H}_{*}\left(S^{n-1}\right)$ must survive in $E_{\infty}$. This section is given by sending a vector $u \in S^{n-1}$ to the unique closed geodesic in $S^{n}$ passing through the marked point $*$ and tangent to $v$. The differentials of the higher pages of the spectral sequence are identically zero for degree reasons. This proves that

$$
\mathbb{H}_{*}\left(\operatorname{Imm}_{*}^{\prime}\left(S^{1}, S^{n}\right)\right):=H_{*+n-1}\left(\operatorname{Imm}_{*}^{\prime}\left(S^{1}, S^{n}\right)\right) \simeq H_{*+n-1}\left(L S^{n-1}\right)=\mathbb{H}_{*}\left(L S^{n-1}\right),
$$

and in fact the isomorphism is given by the 1-jet map. Going back to the Leray-Serre spectral sequence of the fibration (6.1), we have $E_{2}=\Lambda y_{-n+1} \otimes \mathbb{Z}\left[x_{n-2}\right] \otimes \Lambda z_{-n}$. 
Since the differential $d_{2}$ of $E_{2}$ is a derivation, we only need to compute the $d_{2}$ on the generators. It is clear that $d_{2} x_{n-2}=d_{2} y_{n-1}=0$. We claim that $d_{2} z_{n}=0$; this is done by comparison with the spectral sequence of the fibration

$$
\operatorname{Imm}_{*, v}^{\prime}\left(S^{1}, S^{n}\right) \rightarrow \operatorname{Imm}^{\prime}\left(S^{1}, S^{n}\right) \rightarrow U S^{n}
$$

which has a section. Therefore all the elements of $H_{*}\left(U S^{n}\right)$ must survive all the way up to the $E_{\infty}$-page of the Leray-Serre spectral sequence of the fibration (6.2). This implies that $z_{n}$ should survive all the way to the $E_{\infty}$-page, thus $d_{2} z_{n}=0$. For degree reasons we also have $d_{i} z_{n}=0, i>2$. Therefore the $H_{*}\left(\operatorname{Imm}^{\prime}\left(S^{1}, S^{n}\right)\right) \simeq$ $\mathbb{H}_{*}\left(L S^{n-1}\right) \otimes \mathbb{H}_{*}\left(S^{n}\right)$, which proves the statement.

\subsection{Non-triviality of the desingularization morphism}

Let us prove that the desingularization morphism

$$
\phi_{2}: H_{0}\left(\operatorname{Imm}_{2}^{\prime}\left(S^{1}, S^{n}\right)\right) \rightarrow H_{2 n-6}\left(\operatorname{Emb}\left(S^{1}, S^{n}\right)\right)
$$

is non-trivial for $n>4$. By a result of V. Turchin, $H_{2 n-6}\left(\mathrm{Emb}^{l}\left(\mathbb{R}, \mathbb{R}^{n}\right)\right) \simeq \mathbb{Z}$. In [B3, Proposition 3.9], R. Budney proves that $\pi_{2 n-6}\left(\operatorname{Emb}^{l}\left(\mathbb{R}, \mathbb{R}^{n}\right)\right)$, which is the first nontrivial homotopy group. Therefore by Hurewitz's theorem,

$$
\pi_{2 n-6}\left(\operatorname{Emb}^{l}\left(\mathbb{R}, \mathbb{R}^{n}\right)\right) \cong H_{2 n-6}\left(\operatorname{Emb}^{l}\left(\mathbb{R}, \mathbb{R}^{n}\right)\right) \cong \mathbb{Z},
$$

and all elements of $H_{2 n-6}\left(\mathrm{Emb}^{l}\left(\mathbb{R}, \mathbb{R}^{n}\right)\right)$ are spherical. Moreover, he constructs a generator of $\pi_{2 n-6}\left(\mathrm{Emb}^{l}\left(\mathbb{R}, \mathbb{R}^{n}\right)\right) \cong \mathbb{Z}$, which is in the image of the desingularization map ([B3, Theorem 3.13]).

We give a geometric description of this generator: take a long immersion $\gamma: \mathbb{R} \rightarrow$ $\mathbb{R}^{3} \subset \mathbb{R}^{n}$ with two regular double points such that one of its four resolutions in $\mathbb{R}^{3}$ is the trefoil knot. The desingularization morphism maps $\gamma$ to

$$
\text { des: } S^{n-3} \times S^{n-3} \rightarrow \operatorname{Emb}^{l}\left(\mathbb{R}, \mathbb{R}^{n}\right) .
$$

If $s_{2 n-6}$ is the fundamental class of $S^{n-3} \times S^{n-3}$, then in homology des $\operatorname{des}_{*}\left(s_{2 n-6}\right)$ is the generator of $H_{2 n-6}\left(\mathrm{Emb}^{l}\left(\mathbb{R}, \mathbb{R}^{n}\right)\right.$ ) (see [B3, Theorem 3.13]). Let $\gamma^{\prime} \in \operatorname{Imm}_{2}\left(S^{1}, S^{n}\right)$ be a closure of $\gamma$. Using the fact mentioned earlier and the spectral sequence argument, we see that

(1) $H_{2 n-6}\left(\operatorname{Emb}\left(S^{1}, S^{n}\right)\right) \cong \mathbb{Z}$ by using the Serre spectral sequence of the fibration $\operatorname{Emb}\left(S^{1}, S^{n}\right) \rightarrow U S^{n}$ and the fact that this fibration has a cross section we have $H_{2 n-6}\left(\operatorname{Emb}\left(S^{1}, S^{n}\right)\right) \cong E_{2}^{0,2 n-6}$. This element cannot be killed by another class because the existence of a cross section implies that the differential

$$
d_{2 n-5}: H_{2 n-5}\left(U S^{n}\right)=E_{2 n-5}^{2 n-5,0} \rightarrow E_{2 n-5}^{0,2 n-6}=E_{2}^{0,2 n-6} \simeq H_{2 n-6}\left(\operatorname{Emb}^{l}\left(\mathbb{R}, \mathbb{R}^{n}\right)\right)
$$

is identically zero as the zeroth horizontal line has to survive all the way up to $E^{\infty}$-page.

(2) If $k_{2 n-6}$ is a generator of $H_{2 n-6}\left(\operatorname{Emb}\left(S^{1}, S^{n}\right)\right)$, then we have $\phi_{2}\left(1_{\text {tref }}\right)=k_{2 n-6}$ where $1_{\text {tref }}$ is the generator of the 0 -th homology group of the connected component containing $\gamma^{\prime}$. Here we use the naturality of the spectral sequence with respect to the maps between fibration and the fact that in these degrees the desingularization morphism is completely determined by its restriction to the fibers: by the desingularization morphism between long immersions and long knots. 


\section{References}

[Ada] M. Adachi, Embeddings and immersions, Translated from the 1984 Japanese original by Kiki Hudson, Translations of Mathematical Monographs 124, American Mathematical Society, Providence, RI, 1993.

[ALTV] G. Arone, P. Lambrechts, V. Turchin and I. Volić, Coformality and rational homotopy groups of spaces of long knots, Math. Res. Lett. 15 (2008), no. 1, $1-14$.

[Br] J.-L. Brylinski, Loop spaces, characteristic classes and geometric quantization, Progress in Mathematics 107, Birkhäuser Boston Inc., Boston, MA, 1993.

[B1] R. Budney, Little cubes and long knots, Topology 46 (2007), no. 1, 1-27.

[B2] R. Budney, Topology of knot spaces in dimension 3, Proc. Lond. Math. Soc. (3) 101 (2010), no. 2, 477-576.

[B3] R. Budney, A family of embedding spaces, in Geometry, homotopy and configuration spaces Geometry \& Topology Monographs 13 (2008), 41-83.

[BC] R. Budney and F. Cohen, On the homology of the space of knots, Geometry E Topology 13 (2009), no. 1, 99-139.

[BG] J.C. Becker and D.H. Gottlieb, A history of duality in algebraic topology, in History of topology, 725-745, North-Holland, Amsterdam, 1999.

[CCL] A. Cattaneo, P. Cotta-Ramusino and R. Longoni, Configuration spaces and Vassiliev classes in any dimension, Algebr. Geom. Topol. 2 (2002), 949-1000.

[CL] D. Chataur and J.-F. Le Borgne, Homology of spaces of regular loops in the sphere, Algebr. Geom. Topol. 9 (2009), no. 2, 935-977.

[CJY] R.L. Cohen, J.D.S. Jones and J. Yan, The loop-homology algebra of spheres and projective spaces, in Categorical decomposition techniques in algebraic topology (Isle of Skye, 2001), Progress in Mathematics 215 (2004), 77-92, Birkhäuser-Verlag, Basel, 2004.

[CS1] M. Chas and D. Sullivan, String topology, preprint, arXiv math.GT/ 9911159.

[CJ] R. Cohen and J. Jones, A homotopy theoretic realization of string topology, Math. Ann. 324 (2002), no. 4, 773-798.

[GS] K. Gruher and P. Salvatore, Generalized string topology operations, Proc. Lond. Math. Soc. (3) 96 (2008), no. 1, 78-106.

[Hir] M.W. Hirsch, Immersions of manifolds, Trans. Amer. Math. 93 (1959), no. $2,242-276$.

[KS] S. Kallel and S. Salvatore, Rational maps and string topology, Geometry \& Topology 10 (2006), 1579-1606.

[Lau] F. Laudenbach, A note on the Chas-Sullivan product, Enseign. Math. (2) 57 (2011), no. 1-2, 3-21.

[L] J.-F. Le Borgne, The loop-product spectral sequence, Expo. Math. 26 (2008), no. 1, 25-40. 
[S] P. Salvatore, Knots, operads, and double loop spaces, Int. Math. Res. Not. 2006, Art. ID 13628, 22 pp.

[Sm] S. Smale, The classification of immersions of spheres in Euclidean spaces, Ann. of Math. (2) 69 (1959), no. 2, 327-344.

[Tor] V. Tourtchine, On the homology of the spaces of long knots, in Advances in topological quantum field theory, 23-52, NATO Sci. Ser. II Math. Phys. Chem. 179, Kluwer Acad. Publ., Dordrecht, 2004.

[Va] V. Vassiliev, Complements of discriminants of smooth maps: topology and applications, Translations of Mathematical Monographs 98, American Mathematical Society, Providence, RI, 1992.

Hossein Abbaspour abbaspour@univ-nantes.fr

Laboratoire Jean Leray, Université de Nantes, 44322 Nantes, France

David Chataur david.chataur@math.univ-lille1.fr

Laboratoire Paul Painlevé, Université de Lille 1, 59655 Villeneuve d'Ascq Cédex, France 